\title{
32. DATA REPORT: REORIENTATION OF STRUCTURAL FEATURES AT SITES 920 TO 924 USING REMANENT MAGNETIZATION AND MAGNETIC CHARACTERISTICS ${ }^{1}$
}

\author{
Stephen D. Hurst, ${ }^{2}$ Jeff S. Gee, ${ }^{3}$ and Róisín M. Lawrence ${ }^{2}$
}

\begin{abstract}
Drilling at Ocean Drilling Program Sites 920 to 924 recovered core with a diverse set of pervasive structural elements. Site 920 recovered predominantly peridotitic rocks that display an early crystal-plastic fabric overprinted by at least five generations of veins. Sites 921 to 924 recovered gabbros that contain magmatic and metamorphic foliations and lineations developed to varying intensities throughout. Brittle features in the gabbro core include cataclastic zones, faults, and several generations of veins. The characteristic magnetization direction was used to estimate the in situ orientation of structural features within the core. Although significant uncertainty is associated with the unknown effects of anisotropy and tectonic rotations on the remanent declinations, the corrected attitudes of the dominant foliations at Site 920 dip gently east-northeast, parallel to other observations of seafloor structures in the area. Other vein generations and structural features in the rocks do not have a consistent orientation with respect to each other or a consistent variation with core depth. Sites 921-924 were drilled into a section of mostly gabbroic rocks that typically have complicated magnetic properties, with several remanence components identifiable during demagnetization. Reorientation of the gabbro cores is less certain because of the complexity of the remanent magnetization components, however, many structures in the gabbro from Hole $923 \mathrm{~A}$ also seem to have gentle dips to the northeast after such a reorientation.
\end{abstract}

\section{INTRODUCTION}

Ocean Drilling Program (ODP) Sites 920 to 924 are located on the median valley wall of the Mid-Atlantic Ridge near the Kane Transform Zone (MARK area; Fig. 1). The sites are located about $10 \mathrm{~km}$ west of the neovolcanic ridge in the center of the valley. The holes were drilled into serpentinized peridotite (Site 920) and gabbro (Sites 921-924) exposures thought to represent upper mantle and middle to lower oceanic crust. The most prevalent theory for their exposure entails significant faulting and tectonic extension (Karson and Dick, 1983; Karson, 1990; Karson and Lawrence, this volume). The age of these rocks is 0.6 to $0.9 \mathrm{Ma}$, as determined using a $1.3 \mathrm{~cm} / \mathrm{yr}$ halfspreading rate (Schultz et al., 1988; corrected for $0.78 \mathrm{Ma}$ as the age of the Brunhes/Matuyama boundary). The presence of reversed polarity magnetizations at Sites 921-923 indicates a minimum age of $0.78 \mathrm{Ma}$ for these lower crustal gabbros. The uniform normal polarity of the peridotites from Site 920 is consistent with acquisition of the remanent magnetization during the last 0.78 m.y. However, because of the likely role of low-angle extensional faulting in exposing the rocks, the actual age of the rocks remains uncertain. (Bonatti, 1976; Karson and Dick, 1983; Karson, 1990; Cannat, 1993; Cannat et al., 1995). For the peridotite samples, the acquisition of main magnetic remanence likely occurred during serpentinization, although a later thermal event or even the acquisition of a recent viscous remanence is possible. The timing of serpentinization of the peridotites is unknown.

Approximately $325 \mathrm{~m}$ of serpentinized peridotites with subsidiary gabbro and diabase intervals was drilled at Site 920 in two holes (Holes 920B and 920D), $\sim 50 \mathrm{~m}$ apart. Rocks with very similar composition, structure, and mesoscopic fabric were recovered. In general, the dominant lithology is serpentinized harzburgite, which contains a

'Karson, J.A., Cannat, M., Miller, D.J., and Elthon, D. (Eds.), 1997. Proc. ODP, Sci. Results, 153: College Station, TX (Ocean Drilling Program).

${ }^{2}$ Department of Geology, Duke University, Durham, NC 27708, U.S.A. steve@geo.duke.edu

${ }^{3}$ Geosciences Research Division, Scripps Institution of Oceanography, La Jolla, CA 92014 , U.S.A. pervasive mesoscopic foliation defined by elongate relict orthopyroxene grains and subparallel, white, serpentine veins. In much of the core, this foliation has a dip of about $30^{\circ}$. Dunitic intervals generally show fewer white serpentine veins, but commonly show a few elongate relict orthopyroxenes that define the early plastic fabric (Cannat, Karson, Miller, et al., 1995; Ceuleneer and Cannat, this volume). The vast majority of peridotite samples have positive characteristic remanent inclinations in the range $25^{\circ}-45^{\circ}$ (Fig. 2), suggesting magnetization during a single normal polarity interval. The main carrier of the magnetization is magnetite, randomly interspersed within the mesh-textured serpentine and aligned along the edges of serpentine veins (Lawrence et al., this volume).

Four sites (Sites 921-924) were drilled in gabbro exposures to the north of Site 920 , at approximately the same distance from the center of the median valley as Site 920 (Fig. 1). All four sites are located within a radius of $\sim 2 \mathrm{~km}$. Two holes (Holes $921 \mathrm{~A}$ and 923A) were drilled to depths of $80 \mathrm{~m}$ with an average recovery of $50 \%$. The remaining gabbro holes were only drilled to depths of $40 \mathrm{~m}$ or less and had poorer recovery (Cannat, Karson, Miller, et al., 1995).

The gabbros from Sites 921 to 924 include olivine gabbros, metagabbronorites, troctolites, $\mathrm{Fe}-\mathrm{Ti}$ oxide gabbros, and minor volumes of other gabbroic lithology. About $40 \%$ of the gabbros shows evidence of plastic deformation (Shipboard Scientific Party, 1995c, 1995d; Gaggero and Luciano, this volume). The plastic deformation ranges from a subtle foliation and lineation with evident recrystallization of plagioclase in hand specimen to strongly deformed mylonitic zones tens of centimeters thick. A significant proportion of the gabbros $(20 \%)$ consists of lineated and foliated rocks that have lamellar-twinned plagioclase crystals with no undulose extinction or irregular twins indicative of plastic deformation (Shipboard Scientific Party, 1995c, 1995d). The remainder of the core shows no mesoscopic evidence of any penetrative foliation or lineation.

The demagnetization behavior of the gabbro samples is typically much more complex than that of the peridotites, with two and occasionally three or more magnetization components identified (fig. 84 in Shipboard Scientific Party, 1995c). These components have both positive and negative inclinations, indicating that the acquisition of magnetization spanned two or more polarity intervals. Alteration in 
Figure 1. Generalized geologic map of the MARK area, Mid-Atlantic Ridge at $22^{\circ}-24^{\circ} \mathrm{N}$. Contours in $\mathrm{km}$ below sea level.

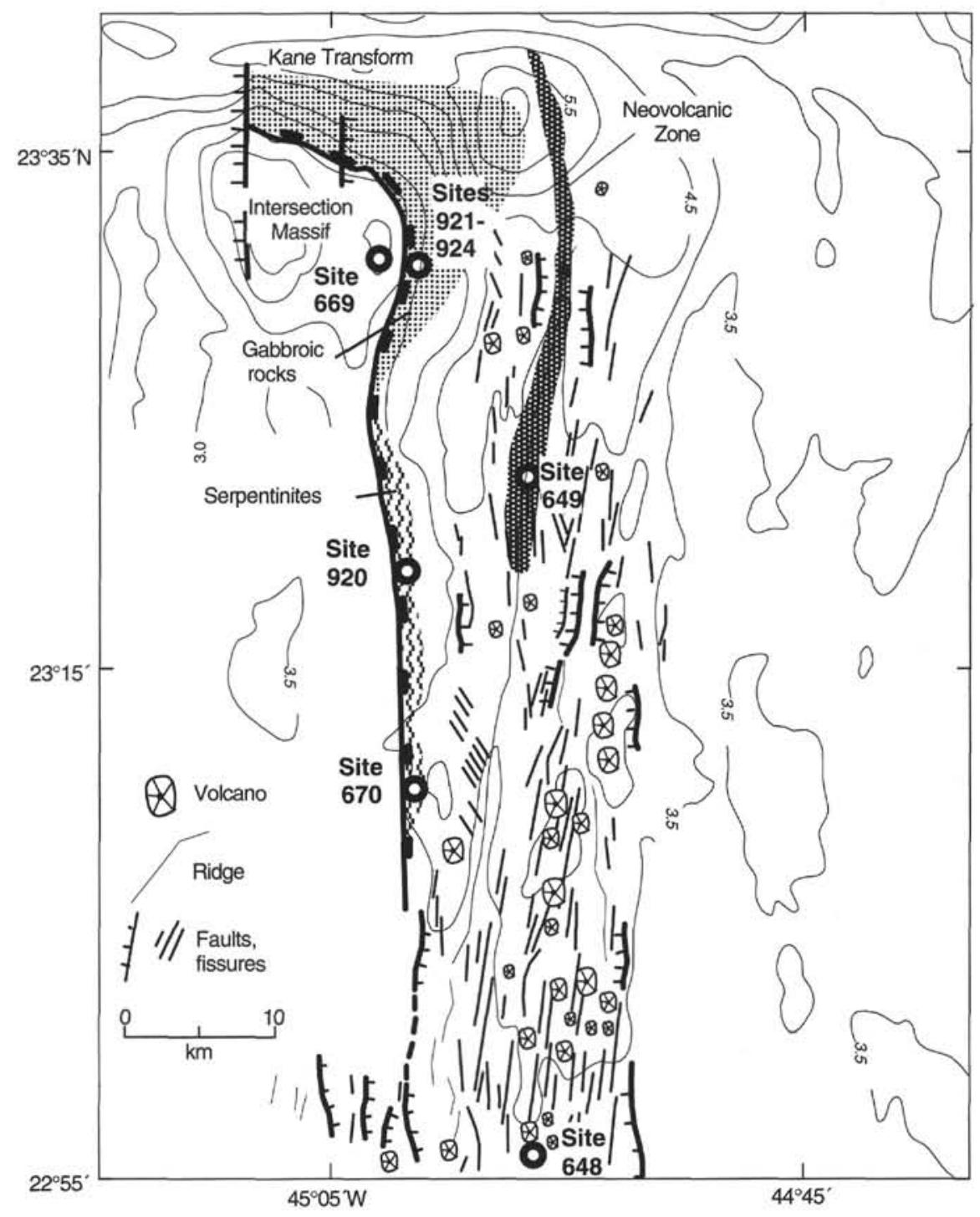

the gabbros may form a spurious, well-defined characteristic magnetization component such as that seen in some of the gabbro samples (Gee et al., this volume). However, some gabbro samples show a single characteristic remanence with an inclination similar to the expected geocentric axial dipole in the area $\left(41^{\circ}\right)$.

\section{REORIENTATION PROCEDURE}

During drilling operations on Leg 153, each piece of core was free to rotate about the vertical axis within the core barrel. No downhole logging was done in any of the holes drilled, therefore the only conceivable means of reorienting the core is using the paleomagnetic demagnetization data (e.g., Cannat and Pariso, 1991). The inclination and declination of the stable remanent magnetic vector were measured in 202 peridotite and 329 gabbro samples (Shipboard Scientific Party, 1995b, 1995d; Gee et al., this volume). Because of the consistent inclination of the characteristic magnetization component in the peridotites, the paleomagnetic vector in each piece of peridotite might, to first order, be inferred to point in the same direction before drilling. The reorientation procedure discussed here applies mainly to peridotite samples (Site 920). However, a small number of structural features from one gabbro site (Hole 923A) are also reoriented using the same procedure.
A total of 153 core pieces or subpieces from Site 920, having either planar or linear structural features measured during shipboard core description, was reoriented using characteristic stable remanence data determined from principle component analysis (Kirschvink, 1980). All the reoriented structural data, including the characteristic declination for Holes 920B and 920D, are reported here, including the data from the 119 core pieces with shipboard paleomagnetic measurements (Tables 1,2). Each reoriented structural feature was in the same piece or subpiece as the paleomagnetic measurement, generally within $15 \mathrm{~cm}$. All of the original measurements were made relative to the ODP core coordinate system (Fig. 3)(Shipboard Scientific Party, 1995a). The shipboard analysis and subsequent shore-based work verified that almost all serpentinized peridotite samples contain a univectorial characteristic magnetization with an average inclination of $35.2^{\circ} \pm 1^{\circ}(\mathrm{N}=202$, the uncertainty is the half angle of the asymmetric $95 \%$ confidence interval, calculated using the inclination-only method of McFadden and Reid [1982]). This average inclination is somewhat less than the inclination $\left(41^{\circ}\right)$ expected for a geocentric axial dipole $(\mathrm{GAD})$ at this site. There are many possible reasons for this discrepancy between the measured average characteristic inclination and the inclination predicted from a $\mathrm{GAD}$ model. One explanation may be that secular variation is inadequately averaged. Because the sole magnetic carrier in the peridotites is magnetite that formed in mesh-textured bulk serpentine as well as 

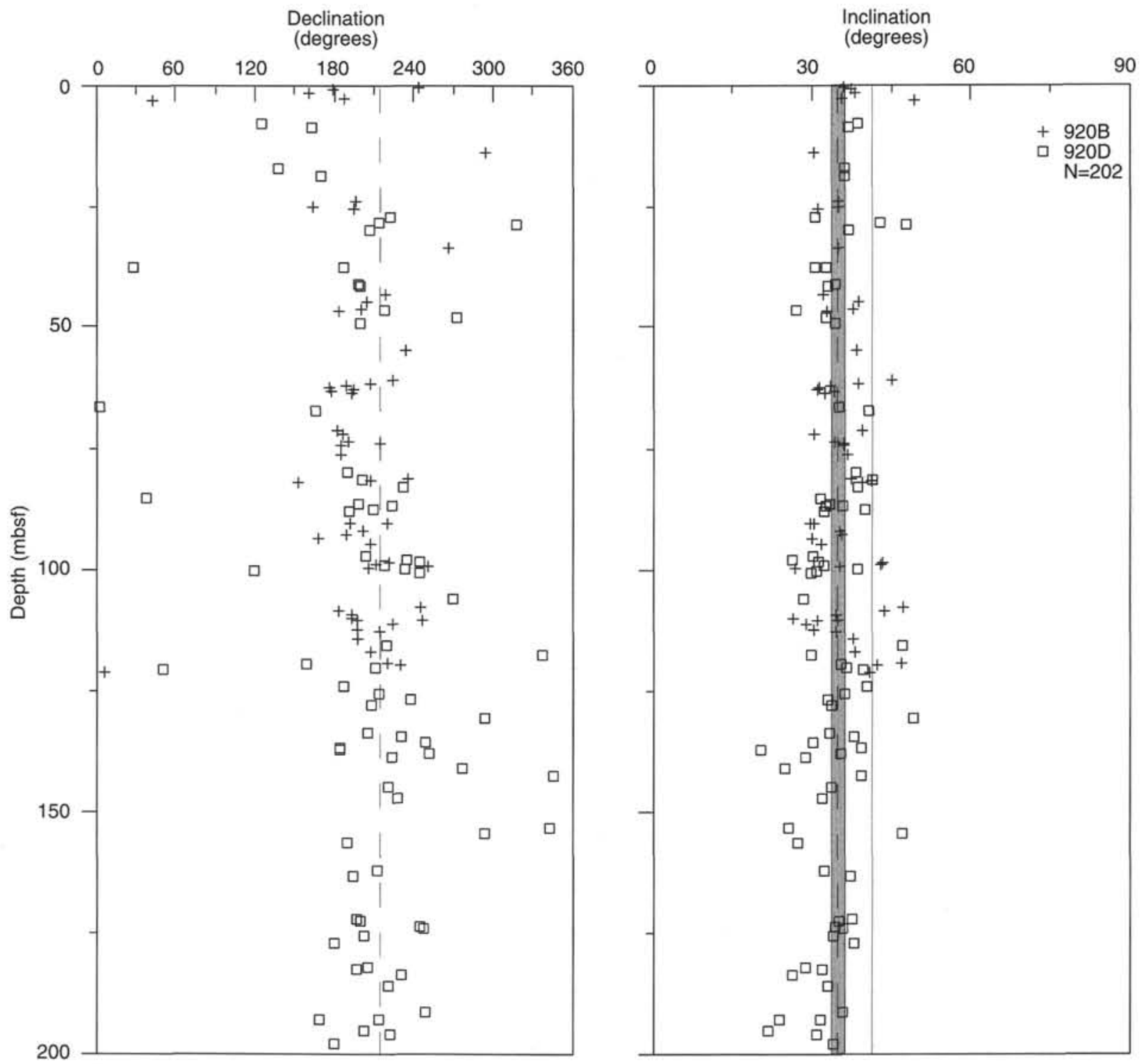

Figure 2. Characteristic remanent declination and inclination vs. depth for 202 discrete minicore samples taken from core from Holes 920B and 920D. Dashed vertical lines show the average value for the combined measurements. The gray box on the inclination plot defines the asymmetric $95 \%$ confidence limits for the mean of the measurements (McFadden and Reid, 1982). The solid vertical line is the expected inclination for a geocentric axial dipole at this latitude $\left(41^{\circ}\right)$.

along crosscutting serpentine veins, the characteristic remanence must have been acquired during or after serpentinization. There are several generations of veins with consistent crosscutting relationships as well as serpentinized remnants of pyroxene and olivine throughout the serpentinized peridotite core. The serpentinization of olivine, pyroxene, and the creation of a succession of serpentine vein sets probably took sufficient time to average secular variation, making inadequate averaging of secular variation an unsatisfactory explanation.

Another possible reason for the discrepancy between the measured inclinations and the expected inclination may be the substantial degree of anisotropy in the magnetic susceptibility (AMS). The AMS is high (average $K_{1} / K_{3}=1.19$ ) in all the peridotite samples measured $(\mathrm{N}=202$ ) (Table 3; Shipboard Scientific Party, 1995b; Lawrence et al., this volume). High degrees of anisotropy of susceptibility, if also indicative of the anisotropy of the remanence-carrying grains, may possibly result in a deflection of remanence acquisition by up to $3^{\circ}$ per 5\% anisotropy (Stacey and Banerjee, 1974). The actual amount of deflection depends upon the angular relationship between the ap- plied field and the orientation of the susceptibility principle axes. Upon reorientation of the characteristic declinations of the peridotites to $0^{\circ}$, the maximum axis $\left(K_{l}\right)$ of the susceptibility ellipsoid is directed to $0^{\circ}$ azimuth with a very shallow plunge, and the minimum axis $\left(K_{3}\right)$ is directed west with approximately a $30^{\circ}$ plunge (Fig. 4). Therefore the direction of $K_{l}$ is typically $25^{\circ}-30^{\circ}$ towards the horizontal from the characteristic magnetization direction. Given the known tectonism at this portion of the Mid-Atlantic Ridge and the expectation that serpentinized peridotites are a lower crustal rock type, another cause of the inclination discrepancy may be rotation of the magnetization by postserpentinization deformation. The majority of faults in the area trend $0^{\circ}-10^{\circ}$; parallel to the axial valley. Assuming that a rotation about a horizontal, north-south axis caused the characteristic inclination to decrease, then a rotation of $\sim 30^{\circ}$ would be needed to change the inclination from $41^{\circ}$ to the observed $35^{\circ}$ (Fig. 5 and Shipboard Scientific Party, 1995b). This rotation would also cause the declination to change from $0^{\circ}$ to $\pm 23^{\circ}$. Rotations about other horizontal axes would need correspondingly less rotation to change the inclination, and would also have proportionally less of an effect on 
Table 1. Reorientation data for samples from Hole 920B.

\begin{tabular}{|c|c|c|c|c|c|c|c|c|c|c|c|c|c|c|c|c|c|}
\hline $\begin{array}{l}\text { Core, } \\
\text { section }\end{array}$ & Piece & $\begin{array}{l}\text { Char. } \\
\text { declin. } \\
\left({ }^{\circ}\right) \\
\text { (uncorr.) }\end{array}$ & Feature & $\begin{array}{c}\text { Strike, dip } \\
\left({ }^{\circ}\right) \\
\text { (uncorr.) }\end{array}$ & $\begin{array}{c}\text { Strike, dip } \\
\left({ }^{\circ}\right) \\
\text { (corr.) }\end{array}$ & $\begin{array}{l}\text { Core, } \\
\text { section }\end{array}$ & Piece & $\begin{array}{l}\text { Char. } \\
\text { declin. } \\
\left({ }^{\circ}\right) \\
\text { (uncorr.) }\end{array}$ & Feature & $\begin{array}{c}\text { Strike, dip } \\
\left({ }^{\circ}\right) \\
\text { (uncorr.) }\end{array}$ & $\begin{array}{c}\text { Strike, dip } \\
\left({ }^{\circ}\right) \\
\text { (corr.) }\end{array}$ & $\begin{array}{l}\text { Core, } \\
\text { section }\end{array}$ & Piece & $\begin{array}{c}\text { Char. } \\
\text { declin. } \\
\left({ }^{\circ}\right) \\
\text { (uncorr.) }\end{array}$ & Feature & $\begin{array}{c}\text { Strike, dip } \\
\left({ }^{\circ}\right) \\
\text { (uncorr.) }\end{array}$ & $\begin{array}{c}\text { Strike, dip } \\
\left({ }^{\circ}\right) \\
\text { (corr.) }\end{array}$ \\
\hline $1 \mathrm{~W}-1$ & 6 & 244 & $\mathrm{Pf}$ & 180,40 & 296,40 & 7R-2 & IB & 176 & V2 & 211,44 & 35,44 & $11 \mathrm{R}-1$ & 2 & 222 & V2 & 190,28 & 328,28 \\
\hline $1 \mathrm{~W}-1$ & 6 & 244 & v3 & 290,79 & 46,79 & $7 \mathrm{R}-2$ & $4 \mathrm{~A}$ & 195 & $\mathrm{~V} 2$ & 222,50 & 27,50 & $11 \mathrm{R}-1$ & 8 & 212 & $\mathrm{v} 2$ & 155,32 & 303,32 \\
\hline iw-2 & 6 & 159 & vo & 211.66 & 52.66 & $7 \mathrm{R}-2$ & 8 & 178 & V2 & 190,50 & 12.50 & IIR-1 & 2 & 222 & $\mathrm{~V} 3 \mathrm{~b}$ & 218,54 & 356,54 \\
\hline IW-2 & i & 181 & V2a & 36,51 & 215,51 & $7 \mathrm{R}-2$ & iB & 176 & V3 & 148,45 & 332,45 & IIR-2 & 1 & 206 & Af & $\begin{array}{l}110,34 \\
180,39\end{array}$ & 334,39 \\
\hline IW-2 & 6 & $\begin{array}{l}101 \\
159\end{array}$ & $\mathrm{~V} 2 \mathrm{a}$ & 186,40 & 27,40 & $7 \mathrm{R}-3$ & 1 & 193 & Af & $\begin{array}{l}1+0,430 \\
190,30\end{array}$ & 357,30 & IIR-2 & 1 & 206 & Pf & 180,39 & 334,39 \\
\hline 1W-3 & 4 & 188 & Pf & 149,48 & 321,48 & $7 \mathrm{R}-3$ & 1 & 193 & $\begin{array}{l}\mathrm{AI} \\
\mathrm{Pf}\end{array}$ & 190,30 & 357,30 & $\begin{array}{l}11 \mathrm{~K}-2 \\
11 \mathrm{R}-2\end{array}$ & 1 & 206 & $\begin{array}{l}\mathrm{P} 1 \\
\mathrm{~V} 2\end{array}$ & $\begin{array}{l}180,39 \\
180,39\end{array}$ & $\begin{array}{l}334, \\
334,39\end{array}$ \\
\hline $1 W-3$ & 6 & 43 & $\mathrm{Pf}$ & 56,46 & 13,46 & $7 \mathrm{R}-3$ & 1 & 193 & V1 & 331,42 & 138,42 & $12 \mathrm{R}-1$ & 2 & 246 & Af & 224,53 & 338,53 \\
\hline $1 \mathrm{~W}-3$ & 4 & 188 & VI & 210,48 & 22,48 & $7 \mathrm{R}-3$ & 1 & 193 & V2 & 190,30 & $\begin{array}{l}150,42 \\
357,30\end{array}$ & $12 \mathrm{R}-1$ & 3 & 183 & Af & 160,20 & 337,20 \\
\hline $1 W-3$ & 6 & 43 & V1 & 42,43 & 359,43 & $8 \mathrm{R}-1$ & 12 & 182 & $\mathrm{Pf}$ & 190,20 & 8,20 & $12 \mathrm{R}-1$ & 3 & 183 & MV & 161,26 & 338,26 \\
\hline $1 \mathrm{~W}-3$ & 6 & 43 & V2 & 132,15 & 89,15 & $8 R-1$ & 12 & 182 & V2 & 189,33 & 7,33 & $12 R-1$ & 2 & 246 & Pf & 177,37 & 291,37 \\
\hline $1 \mathrm{~W}-3$ & 4 & 188 & $\mathrm{~V} 2$ & 140,76 & 312,76 & $8 \mathrm{R}-2$ & 6 & 187 & Af & 174,38 & 347,38 & $12 R-1$ & 3 & 183 & Pf & 167,33 & 344,33 \\
\hline $1 \mathrm{~W}-3$ & 4 & 188 & V3 & 135,60 & 307,60 & $8 \mathrm{R}-2$ & 6 & 187 & vo & 79,15 & 252,15 & $12 \mathrm{R}-1$ & 2 & 246 & voa & 303,34 & 57,34 \\
\hline $2 \mathrm{R}-1$ & 2 & 294 & V2 & 338,40 & 44,40 & $8 \mathrm{R}-2$ & 6 & 187 & V2 & 169,36 & 342,36 & $12 \mathrm{R}-1$ & 2 & 246 & vob & 265,31 & 19,31 \\
\hline $2 \mathrm{R}-1$ & 2 & 294 & V3 & 343,72 & 49,72 & $8 \mathrm{R}-3$ & 11 & 214 & Af & 180,40 & 326,40 & $12 R-1$ & 2 & 246 & $\begin{array}{l}\text { V2 } \\
\text { Vo }\end{array}$ & 183,32 & 297,32 \\
\hline $3 R-1$ & 5 & 196 & Af & 175,25 & 339,25 & $8 \mathrm{R}-3$ & II & 214 & Pf & 180,40 & $\begin{array}{l}320,40 \\
326,40\end{array}$ & $12 \mathrm{R}-1$ & 3 & $\begin{array}{l}240 \\
183\end{array}$ & v2 & 160,20 & 337,20 \\
\hline $3 R-1$ & 5 & 196 & Pf & 175,20 & 33920 & $8 \mathrm{R}-3$ & 7 & 190 & vo & $\begin{array}{l}180,30 \\
190,32\end{array}$ & $\begin{array}{l}320,40 \\
0,32\end{array}$ & $12 \mathrm{R}-1$ & 2 & $\begin{array}{l}183 \\
246\end{array}$ & $\mathrm{~V} 3$ & $\begin{array}{l}100,20 \\
127,74\end{array}$ & $\begin{array}{l}331,70 \\
241,74\end{array}$ \\
\hline $\begin{array}{l}3 \mathrm{~K}-1 \\
3 \mathrm{R}-1\end{array}$ & 5 & $\begin{array}{l}190 \\
196\end{array}$ & $\begin{array}{l}\mathrm{P}_{1} \\
\mathrm{~V} 2\end{array}$ & 175,25 & $\begin{array}{l}339,20 \\
339,25\end{array}$ & $\begin{array}{l}8 \mathrm{~K}-3 \\
8 \mathrm{R}-3\end{array}$ & 11 & $\begin{array}{l}190 \\
214\end{array}$ & vo & $\begin{array}{l}190,32,32 \\
198\end{array}$ & $\begin{array}{l}0,32 \\
344,32\end{array}$ & $\begin{array}{l}12 K-1 \\
12 R-1\end{array}$ & 3 & $\begin{array}{l}240 \\
183\end{array}$ & $\begin{array}{l}\sqrt{3} \\
\text { V3 }\end{array}$ & 241,76 & $\begin{array}{l}241,74 \\
58,76\end{array}$ \\
\hline $3 R-1$ & 5 & 196 & v3 & 102,90 & 266,90 & $8 \mathrm{R}-3$ & 7 & $\begin{array}{l}194 \\
190\end{array}$ & v2 & 184,42 & 354,42 & $12 \mathrm{R}-2$ & 4 & 194 & Af & 173,42 & 339,42 \\
\hline $3 \mathrm{R}-2$ & 10 & 194 & Af & 170,45 & $\begin{array}{l}200,900 \\
336,45\end{array}$ & $\begin{array}{l}8 \mathrm{~K}-3 \\
8 \mathrm{R}-3\end{array}$ & 11 & $\begin{array}{l}100 \\
214\end{array}$ & $\begin{array}{l}\mathrm{V} 2 \\
\mathrm{~V} 2\end{array}$ & $\begin{array}{l}184,42 \\
180,40\end{array}$ & $\begin{array}{l}35,42 \\
326,40\end{array}$ & $12 \mathrm{R}-2$ & $\begin{array}{l}4 \\
5\end{array}$ & $\begin{array}{l}194 \\
193\end{array}$ & $\begin{array}{l}\text { AI } \\
\text { Af }\end{array}$ & $\begin{array}{l}167,52 \\
\end{array}$ & $\begin{array}{l}339,42 \\
334,55\end{array}$ \\
\hline $3 R-2$ & 4 & $\begin{array}{l}194 \\
164\end{array}$ & $\begin{array}{l}\text { AI } \\
\text { Pf }\end{array}$ & $\begin{array}{r}169,45 \\
\end{array}$ & $\begin{array}{l}336,48 \\
5,48\end{array}$ & $\begin{array}{l}8 \mathrm{k}-3 \\
8 \mathrm{R}-4\end{array}$ & 2 & $\begin{array}{l}214 \\
185\end{array}$ & $\begin{array}{l}\mathrm{V} \\
\mathrm{Pf}\end{array}$ & $\begin{array}{l}180,40 \\
169,25\end{array}$ & $\begin{array}{l}320,40 \\
344,25\end{array}$ & $12 \mathrm{R}-2$ & 6 & 247 & Af & 15,34 & 128,34 \\
\hline $3 \mathrm{R}-2$ & 10 & 194 & $\mathrm{Pf}$ & 162,46 & 328,46 & $8 \mathrm{R}-4$ & 2 & 185 & voa & 15,61 & 190,61 & $12 \mathrm{R}-2$ & 4 & 194 & CL1 & 170,16 & $\begin{array}{l}128,54 \\
336,16\end{array}$ \\
\hline $3 \mathrm{R}-2$ & 4 & 164 & V1 & 168,82 & 4,82 & $8 \mathrm{R}-4$ & 2 & 185 & V2 & 187,34 & 2,34 & $12 R-2$ & 4 & 194 & CL2 & 232,52 & 38,52 \\
\hline $3 R-2$ & 10 & 194 & vi & 172,27 & 338,27 & $8 \mathrm{R}-5$ & 5 & 185 & vo & 202,33 & 17,33 & $12 \mathrm{R}-2$ & 6 & 247 & $\mathrm{FI}$ & 284,33 & 37,33 \\
\hline $3 \mathrm{R}-2$ & 4 & 164 & V2 & 177.38 & 13,38 & $8 \mathrm{R}-5$ & 5 & 185 & $\mathrm{~V} 2$ & 192,31 & 7,31 & $12 \mathrm{R}-2$ & 6 & 247 & $\mathrm{Fl}$ & L:32/310 & $\mathrm{L}: 32 / 197$ \\
\hline $3 \mathrm{R}-2$ & 10 & 194 & $\mathrm{~V} 2$ & 170,45 & 336,45 & $9 \mathrm{R}-2$ & 5 & 152 & Af & 102,70 & 310,70 & $12 \mathrm{R}-2$ & 6 & 247 & F2 & 277,68 & 30.68 \\
\hline $4 \mathrm{R}-1$ & 7 & 266 & Af & 180,25 & 274,25 & $9 \mathrm{R}-2$ & 5 & 152 & $\mathrm{Pf}$ & 102,70 & 310,70 & $12 \mathrm{R}-2$ & 6 & 247 & $\mathrm{~F}_{2}$ & L.42/302 & $\begin{array}{l}3.00 \\
\mathrm{~L}-42 / 189\end{array}$ \\
\hline $4 \mathrm{R}-1$ & 7 & 266 & $\mathrm{Pf}$ & 180,25 & 274,25 & $9 \mathrm{R}-2$ & 5 & 152 & V1 & 102,70 & 310,70 & $12 \mathrm{R}-2$ & 5 & 193 & $\mathrm{Pf}$ & 158,5 & 325,5 \\
\hline $4 R-1$ & 7 & 266 & V2 & 180,25 & 274,25 & $9 \mathrm{R}-2$ & 4 & 207 & V1a & 195,82 & 348,82 & $12 \mathrm{R}-2$ & 4 & 194 & V1 & 255,26 & 61.26 \\
\hline $\begin{array}{l}4 R-1 \\
5 R-2\end{array}$ & 10 & 200 & Af & 180,40 & $\begin{array}{l}274,23 \\
335,40\end{array}$ & $9 \mathrm{R}-2$ & $\begin{array}{l}4 \\
4\end{array}$ & 207 & $\begin{array}{l}\text { VIa } \\
\text { V1b }\end{array}$ & $\begin{array}{l}193,82 \\
229,11\end{array}$ & $\begin{array}{l}348,11 \\
22,11\end{array}$ & $\begin{array}{l}12 \mathrm{R}-2 \\
12 \mathrm{R}-2\end{array}$ & $\begin{array}{l}4 \\
6\end{array}$ & $\begin{array}{l}194 \\
247\end{array}$ & $\begin{array}{l}v_{1} \\
\text { vi }\end{array}$ & $\begin{array}{l}253,20 \\
33,57\end{array}$ & $\begin{array}{l}01,20 \\
146,57\end{array}$ \\
\hline $\begin{array}{l}5 R-2 \\
5 R-2\end{array}$ & $\begin{array}{l}10 \\
10\end{array}$ & 205 & $\begin{array}{l}\mathrm{AI} \\
\mathrm{Pf}\end{array}$ & $\begin{array}{l}180,40 \\
180,40\end{array}$ & $\begin{array}{l}335,40 \\
335,40\end{array}$ & $\begin{array}{l}9 \mathrm{~K}-2 \\
9 \mathrm{R}-2\end{array}$ & $\begin{array}{l}4 \\
1\end{array}$ & $\begin{array}{l}201 \\
236\end{array}$ & $\begin{array}{l}\text { V1b } \\
\text { V2 }\end{array}$ & $\begin{array}{l}229,111 \\
189,44\end{array}$ & $\begin{array}{l}22,11 \\
313,44\end{array}$ & $\begin{array}{l}12 \mathrm{R}-2 \\
12 \mathrm{R}-2\end{array}$ & $\begin{array}{l}0 \\
4\end{array}$ & $\begin{array}{l}241 \\
194\end{array}$ & $\begin{array}{l}\mathrm{v} 1 \\
\mathrm{~V} 2\end{array}$ & $\begin{array}{l}33,31 \\
173,42\end{array}$ & $\begin{array}{l}140,31 \\
339,42\end{array}$ \\
\hline $5 R-2$ & 10 & 205 & $\mathrm{v} 2$ & 180,37 & 335,37 & $9 \mathrm{R}-2$ & 3 & 236 & $\mathrm{v} 2$ & 189,44 & 313,44 & $12 \mathrm{R}-2$ & 5 & 193 & $\mathrm{v} 2$ & 167.55 & $\begin{array}{l}339,42 \\
334.55\end{array}$ \\
\hline $6 \mathrm{R}-3$ & 2 & 234 & $\mathrm{Pf}$ & 120,59 & 246,59 & $9 \mathrm{R}-2$ & 4 & 207 & $\begin{array}{l}\mathrm{V} 2 \\
\mathrm{~V} 2\end{array}$ & $\begin{array}{l}189,44 \\
180,15\end{array}$ & 333,15 & $12 \mathrm{R}-2$ & $\begin{array}{l}5 \\
6\end{array}$ & 247 & $\begin{array}{l}\mathrm{V} 2 \\
\mathrm{~V} 2\end{array}$ & $\begin{array}{l}107,55 \\
13,5\end{array}$ & $\begin{array}{l}334,53 \\
126,55\end{array}$ \\
\hline $6 \mathrm{R}-3$ & 2 & $\begin{array}{l}234 \\
234\end{array}$ & $\begin{array}{l}11 \\
\text { V2 }\end{array}$ & $\begin{array}{l}147.59 \\
\end{array}$ & 273,51 & $9 \mathrm{R}-2$ & ${ }_{5}^{4}$ & 152 & $\mathrm{v} 2$ & 23066 & 78,66 & $12 \mathrm{R}-3$ & 8 & 224 & Af & 192.43 & 328,43 \\
\hline $6 \mathrm{R}-3$ & 2 & 234 & V3 & 282,18 & 48,18 & $9 \mathrm{R}-2$ & 5 & 152 & V3 & 184,77 & $\begin{array}{l}18,00 \\
32,77\end{array}$ & $12 \mathrm{R}-3$ & 3 & 197 & $\begin{array}{l}\text { AI } \\
\text { V2 }\end{array}$ & $\begin{array}{l}192,43 \\
184,68\end{array}$ & $\begin{array}{l}328,43 \\
347,68\end{array}$ \\
\hline $7 \mathrm{R}-1$ & $3 \mathrm{~B}$ & 224 & Af & 257,77 & 33,77 & $10 R-2$ & 1 & 192 & Af & 153,27 & 321,27 & $12 R-3$ & 8 & 224 & $\mathrm{v} 2$ & 192,43 & 328,43 \\
\hline $7 \mathrm{R}-1$ & $4 \mathrm{~A}$ & 207 & Af & 222,65 & 15,65 & $10 \mathrm{R}-2$ & 2 & 220 & Af & 205,43 & 345,43 & $12 R-4$ & 4 & 197 & V2 & 173,59 & 336,59 \\
\hline $7 \mathrm{R}-1$ & $5 \mathrm{~B}$ & 189 & Af & 142,43 & 313,43 & $10 \mathrm{R}-2$ & 2 & 220 & Pf & 205,43 & 345,43 & $12 R-5$ & $1 \vec{A}$ & 214 & Af & 129,64 & 275,64 \\
\hline $7 \mathrm{R}-1$ & $3 \mathrm{~A}$ & 224 & $\mathrm{CL}$ & 230,54 & 6,54 & $10 \mathrm{R}-2$ & 1 & 192 & V2 & 153,27 & 321,27 & $12 \mathrm{R}-5$ & IA & 214 & V2 & 129,64 & 275,64 \\
\hline $7 \mathrm{R}-1$ & $5 \mathrm{~A}$ & 189 & CL & 144,30 & 315,30 & $10 \mathrm{R}-2$ & 2 & 220 & V2 & 205,43 & 345,43 & $12 \mathrm{R}-5$ & IB & $\begin{array}{l}214 \\
214\end{array}$ & V $3 b$ & $\begin{array}{l}179,04 \\
L: 42 / 270\end{array}$ & $\mathrm{~L}: 42 / 124$ \\
\hline $7 \mathrm{R}-1$ & $3 \mathrm{E}$ & 224 & $\mathrm{Pf}$ & 165,47 & 301,47 & $10 R-2$ & 2 & 220 & $\mathrm{~V} 3$ & 200,81 & 340,81 & $12 \mathrm{R}-6$ & 1 & 197 & $\begin{array}{l}\text { Afi } \\
\text { Ant }\end{array}$ & 176,37 & 339.37 \\
\hline 7R-1 & $4 \mathrm{~A}$ & 207 & $\mathrm{Pf}$ & 194.60 & 347.60 & $10 \mathrm{R}-3$ & 5 & 202 & Af & $\begin{array}{l}200,81 \\
180,40\end{array}$ & $\begin{array}{l}340,81 \\
338,40\end{array}$ & $\begin{array}{l}12 R-6 \\
12 R-6\end{array}$ & 1 & $\begin{array}{l}197 \\
197\end{array}$ & Af2 & $\begin{array}{l}1 / 10,37 \\
152,28\end{array}$ & $\begin{array}{l}339,31 \\
315,28\end{array}$ \\
\hline $\begin{array}{l}7 \mathrm{~K}-1 \\
7 \mathrm{R}-1\end{array}$ & $\begin{array}{l}4 \mathrm{~A} \\
5 \mathrm{~B}\end{array}$ & $\begin{array}{l}201 \\
189\end{array}$ & $\begin{array}{l}\mathrm{PI} \\
\mathrm{Pf}\end{array}$ & $\begin{array}{r}194,60 \\
209,42\end{array}$ & $\begin{array}{l}34,600 \\
20,42\end{array}$ & $\begin{array}{l}10 \mathrm{~K}-3 \\
10 \mathrm{R}-3\end{array}$ & 8 & $\begin{array}{l}202 \\
189\end{array}$ & $\begin{array}{l}\text { AI } \\
\text { Af }\end{array}$ & $\begin{array}{l}180,40 \\
180,35\end{array}$ & $\begin{array}{l}358,40 \\
351,35\end{array}$ & $12 \mathrm{R}-6$ & 1 & 197 & $\begin{array}{l}\text { A12 } \\
\text { MV }\end{array}$ & $\begin{array}{l}132,28 \\
245,48\end{array}$ & $\begin{array}{l}315,28 \\
48,48\end{array}$ \\
\hline $\begin{array}{l}7 \mathrm{R}-1 \\
7 \mathrm{R}-1\end{array}$ & $\begin{array}{l}5 \mathrm{~B} \\
4 \mathrm{~B}\end{array}$ & $\begin{array}{l}189 \\
207\end{array}$ & $\begin{array}{l}\text { Pf } \\
\text { v0 }\end{array}$ & $\begin{array}{l}209,42 \\
31,49\end{array}$ & $\begin{array}{l}20,42 \\
184,49\end{array}$ & $\begin{array}{l}10 \mathrm{R}-3 \\
10 \mathrm{R}-3\end{array}$ & $\begin{array}{l}8 \\
5\end{array}$ & $\begin{array}{l}189 \\
202\end{array}$ & $\begin{array}{l}\text { At } \\
\text { Pf }\end{array}$ & $\begin{array}{l}180,35 \\
180,40\end{array}$ & $\begin{array}{l}351,35 \\
338,40\end{array}$ & $\begin{array}{l}12 R-6 \\
12 R-6\end{array}$ & 1 & $\begin{array}{l}191 \\
197\end{array}$ & $\begin{array}{l}\text { MV } \\
\text { MV }\end{array}$ & $\begin{array}{l}245,48 \\
138,31\end{array}$ & $\begin{array}{l}48,48 \\
301.31\end{array}$ \\
\hline 7R-1 & $3 \mathrm{~B}$ & 224 & v2 & 257,77 & 33,77 & $10 R-3$ & 8 & $\begin{array}{l}202 \\
189\end{array}$ & $\begin{array}{l}\mathrm{PT} \\
\mathrm{Pf}\end{array}$ & $\begin{array}{l}180,40 \\
180.35\end{array}$ & $\begin{array}{l}358,40 \\
351,35\end{array}$ & $12 R-6$ & 1 & 197 & Pf & 162,40 & $\begin{array}{l}301,31 \\
325,40\end{array}$ \\
\hline $7 \mathrm{R}-1$ & $4 \mathrm{~A}$ & 207 & V2 & 231,63 & 24,63 & $10 \mathrm{R}-3$ & 8 & 189 & V1 & 180,35 & 351,35 & $12 \mathrm{R}-6$ & 1 & 197 & $\begin{array}{l}\mathrm{r} 1 \\
\mathrm{~V} 2\end{array}$ & 176,37 & 339,37 \\
\hline $7 \mathrm{R}-1$ & $4 \mathrm{~B}$ & 207 & V2 & $\begin{array}{l}231,73 \\
189,77\end{array}$ & $\begin{array}{l}24,03 \\
342,77\end{array}$ & $10 \mathrm{R}-3$ & 8 & $\begin{array}{l}189 \\
189\end{array}$ & $\begin{array}{l}\text { V2 } \\
\text { V }\end{array}$ & 180,70 & 351,70 & $13 \mathrm{R}-1$ & 2 & 208 & Af & $\begin{array}{l}170,37 \\
140,36\end{array}$ & 292,36 \\
\hline $7 \mathrm{R}-1$ & $5 \mathrm{~B}$ & 18 & V2 & 142,43 & 313,43 & $10 R$ & 5 & 202 & V2a & 180,55 & 338,55 & $13 R-1$ & 2 & 208 & $\mathrm{Pf}$ & $\begin{array}{l}140,36 \\
140,36\end{array}$ & $\begin{array}{l}292,36 \\
292,36\end{array}$ \\
\hline $7 \mathrm{R}-1$ & $3 \mathrm{~B}$ & 224 & V3 & 135,58 & 271,58 & $10 R-3$ & 8 & 189 & V3 & 180,90 & 351,90 & $13 R-1$ & 2 & 208 & V1 & 270,18 & 62,18 \\
\hline $7 \mathrm{R}-1$ & $4 \mathrm{~A}$ & 207 & v3 & 223,78 & $16,78^{\circ}$ & $10 R-4$ & 2 & 168 & Af & 188,45 & 20.45 & $13 R-1$ & 2 & 208 & $\mathrm{v} 2$ & 140,36 & 292,36 \\
\hline $7 \mathrm{R}-1$ & $5 \mathrm{~B}$ & 189 & V3 & 201,90 & 12,90 & $10 R-4$ & 2 & 168 & Pf & 188,45 & 20,45 & $13 R-2$ & 7 & 220 & Af & $\begin{array}{l}140,50 \\
180.28\end{array}$ & 320.28 \\
\hline $7 \mathrm{R}-2$ & $1 \mathrm{~B}$ & 176 & Af & 145,57 & 32957 & $10 R-4$ & 2 & 168 & v2 & 188,45 & 20,45 & $13 R-3$ & IA & 230 & Af & 150,40 & 280,40 \\
\hline $7 \mathrm{R}-2$ & $4 \mathrm{~A}$ & 195 & Af & 222,50 & 27,50 & $10 \mathrm{R}-5$ & 1 & 206 & $\mathrm{Pf}$ & 191,44 & 345,44 & 13R-3 & iA & 230 & $\mathrm{Pf}$ & 170,42 & $\begin{array}{l}280,40 \\
300,42\end{array}$ \\
\hline $7 \mathrm{R}-2$ & $\begin{array}{l}4 A \\
8\end{array}$ & 178 & Af & 19050 & 1250 & $10 R-5$ & 1 & 206 & $\mathrm{Y}^{1}$ & 180.40 & 334,40 & $13 R-3$ & IA & 230 & $\mathrm{y}_{1}$ & 15.71 & $\begin{array}{l}300,42 \\
145,71\end{array}$ \\
\hline $7 \mathrm{R}-2$ & iB & 176 & MY & 50.54 & 23454 & $11 \mathrm{R}-1$ & 1 & 200 & $1 \mathrm{MV}$ & $\begin{array}{l}100,40 \\
168.32\end{array}$ & $\begin{array}{l}534,40 \\
277,3\end{array}$ & 3R-3 & A & 230 & $\mathrm{y}_{2}$ & 15040 & 280,40 \\
\hline $7 \mathrm{R}-2$ & IB & 176 & Pf & 143.56 & $\begin{array}{l}234,54 \\
32756\end{array}$ & IIR-1 & $\frac{1}{2}$ & 202 & Pf & 100,52 & 328,28 & $3 R-4$ & 3 & 57 & DI & 100, 70 & $\begin{array}{l}280,40 \\
2007\end{array}$ \\
\hline $\begin{array}{l}7 \mathrm{~K}-2 \\
7 \mathrm{R}-2\end{array}$ & $\begin{array}{l}1 \mathrm{~B} \\
4 \mathrm{~A}\end{array}$ & 195 & Pf & $\begin{array}{l}143,50 \\
219,54\end{array}$ & $\begin{array}{l}32,5,56 \\
24,54\end{array}$ & $\begin{array}{l}11 K-1 \\
11 R-1\end{array}$ & 8 & 212 & Pf & $\begin{array}{l}190,28 \\
155,32\end{array}$ & $\begin{array}{l}328,28 \\
303,32\end{array}$ & $13 R-4$ & 3 & 5.7 & $\begin{array}{l}\mathrm{D} 1 \\
\mathrm{Pf}\end{array}$ & $\begin{array}{l}200,12 \\
175,70\end{array}$ & $\begin{array}{l}200,72 \\
169,70\end{array}$ \\
\hline $7 \mathrm{R}-2$ & $\begin{array}{c}4 A \\
8\end{array}$ & 178 & Pf & 165,35 & 347,35 & $11 \mathrm{R}-1$ & 14 & 251 & Pf & 168,32 & 277,32 & & & & & & \\
\hline
\end{tabular}

Notes: The correction factor is equal to $360^{\circ}-\mathrm{D}$ (the uncorrected stable remanent declination). Therefore the corrected orientations are in the system where north is parallel to the remanent declination. Char. declin. = characteristic declination, uncorr. = uncorrected, corr. = corrected. Feature abbreviations are described in Shipboard Scientific Party (1995a) and in other portions of this volume (Dilek, Coulton, et al., this volume). 
Table 2. Reorientation data for samples from Hole 920D.

\begin{tabular}{|c|c|c|c|c|c|c|c|c|c|c|c|c|c|c|c|c|c|c|c|c|c|c|c|}
\hline $\begin{array}{l}\text { Core, } \\
\text { section }\end{array}$ & Piece & $\begin{array}{l}\text { Char. } \\
\text { declin. } \\
\left.\text { ( }{ }^{\circ}\right) \\
\text { (uncorr.) }\end{array}$ & Feature & $\begin{array}{c}\text { Strike, dip } \\
\left({ }^{\circ}\right) \\
\text { (uncorr.) }\end{array}$ & $\begin{array}{l}\text { Strike, dip } \\
\left({ }^{\circ}\right) \\
\text { (corr.) }\end{array}$ & $\begin{array}{l}\text { Core, } \\
\text { section }\end{array}$ & Piece & $\begin{array}{l}\text { Char. } \\
\text { declin. } \\
\left({ }^{\circ}\right) \\
\text { (uncorr.) }\end{array}$ & Feature & $\begin{array}{c}\text { Strike, dip } \\
\left({ }^{\circ}\right) \\
\text { (uncorr.) }\end{array}$ & $\begin{array}{l}\text { Strike, dip } \\
\left.\text { ( }{ }^{\circ}\right) \\
\text { (corr.) }\end{array}$ & $\begin{array}{l}\text { Core, } \\
\text { section }\end{array}$ & Piece & $\begin{array}{c}\text { Stable } \\
\text { declin. } \\
\left({ }^{\circ}\right) \\
\text { (uncorr.) }\end{array}$ & Feature & $\begin{array}{c}\text { Strike, dip } \\
\left({ }^{\circ}\right) \\
\text { (uncorr.) }\end{array}$ & $\begin{array}{l}\text { Strike, dip } \\
\left({ }^{\circ}\right) \\
\text { (corr.) }\end{array}$ & $\begin{array}{l}\text { Core, } \\
\text { section }\end{array}$ & Piece & $\begin{array}{l}\text { Char. } \\
\text { declin. } \\
\left({ }^{\circ}\right) \\
\text { (uncorr.) }\end{array}$ & Feature & $\begin{array}{c}\text { Strike, dip } \\
\left({ }^{\circ}\right) \\
\text { (uncorr.) }\end{array}$ & $\begin{array}{c}\text { Strike, dip } \\
\left({ }^{\circ}\right) \\
(\text { corr.) }\end{array}$ \\
\hline $2 \mathrm{R}-1$ & 4 & 126 & V3 & 170,36 & 44,36 & $11 \mathrm{R}-1$ & 4 & 38 & v0 & 182,90 & 144,90 & $14 R-5$ & 7 & 52 & Af & 344,68 & 292,68 & $20 \mathrm{R}-2$ & $1 \mathrm{~A}$ & 201 & V2 & 141,6 & 300,6 \\
\hline $2 \mathrm{R}-1$ & 4 & 126 & v1 & 318,67 & 192,67 & $11 \mathrm{R}-1$ & 4 & 38 & VI & 183,56 & 145,56 & $14 \mathrm{R}-5$ & 7 & 52 & $\mathrm{Pf}$ & 344,68 & 292,68 & $20 \mathrm{R}-2$ & IB & 201 & Pf & 220,37 & 19,37 \\
\hline $2 \mathrm{R}-1$ & 4 & 126 & $\mathrm{~V} 4$ & 125,47 & 359,47 & $11 \mathrm{R}-1$ & 4 & 38 & $\mathrm{Pf}$ & 11,46 & 333,46 & $14 R-5$ & 7 & 52 & V2a & 344,68 & 292,68 & $20 \mathrm{R}-3$ & 1 & 246 & V1a & 187,22 & 301,22 \\
\hline $2 \mathrm{R}-1$ & 13 & 164 & vo & 223,17 & 59,17 & $11 \mathrm{R}-1$ & 13 & 199 & $\mathrm{Pf}$ & 157,51 & 318,51 & $15 \mathrm{R}-1$ & 4 & 188 & VId & 192,80 & 4,80 & $20 \mathrm{R}-3$ & 1 & 246 & V 22 & 30,44 & 344,44 \\
\hline $2 \mathrm{R}-\mathrm{i}$ & 13 & 164 & $\mathrm{Pf}$ & 132,67 & 328,67 & $11 \mathrm{R}-1$ & 13 & 199 & V2 & 160,50 & 321,50 & $15 R-1$ & 4 & 188 & Vle & 2,48 & 174,48 & 20R-3 & 1 & 246 & V $1 \mathrm{~b}$ & 0,78 & 114,78 \\
\hline $3 R-1$ & 3 & 138 & Af & 165,45 & 27,45 & $11 \mathrm{R}-2$ & 3 & 224 & V2 & 167,41 & 303,41 & $15 \mathrm{R}-1$ & 4 & 188 & Vla & 3,49 & 175,49 & $20 \mathrm{R}-3$ & $2 \mathrm{~A}$ & 248 & V2 & 269,37 & 21,37 \\
\hline $3 R-1$ & 3 & 138 & Pf & 165,45 & 27,45 & IIR-2 & 3 & 224 & Vlb & 189,80 & 325,80 & $15 \mathrm{R}-1$ & 4 & 188 & V2 & 177,55 & 349,55 & $20 \mathrm{R}-3$ & $2 \mathrm{~A}$ & 248 & F2 & 83,57 & 35,57 \\
\hline $3 R-1$ & 3 & 138 & $\mathrm{~V} 2$ & 165,45 & 27,45 & $11 \mathrm{R}-2$ & 4 & 224 & Pf & 172,48 & 308,48 & $15 \mathrm{R}-1$ & 4 & 188 & Vlb & 0,17 & 172,17 & $20 \mathrm{R}-3$ & $2 \mathrm{~A}$ & 248 & & L:357/40 & L: $109 / 40$ \\
\hline $3 \mathrm{R}-1$ & 3 & 138 & $\sqrt{3}$ & 350,58 & 212,58 & $11 \mathrm{R}-2$ & 4 & 224 & $\mathrm{~V} 2$ & 172,48 & 308,48 & $15 \mathrm{R}-2$ & 3 & 214 & V1 & 12,87 & 158,87 & $20 \mathrm{R}-3$ & $2 \mathrm{~A}$ & 248 & V3 & 225,15 & 337,15 \\
\hline $3 \mathrm{R}-2$ & 4 & 171 & Af & 251,15 & 80,15 & $11 \mathrm{R}-2$ & 4 & 224 & VIb & 180,93 & 16,9 & $15 \mathrm{R}-2$ & 3 & 214 & $\mathrm{~V} 2$ & 171,28 & 317,28 & $20 \mathrm{R}-3$ & $2 \mathrm{C}$ & 248 & Vla & 212,86 & 324,86 \\
\hline $3 \mathrm{R}-2$ & 4 & 171 & $\mathrm{Pf}$ & 251,15 & 80,15 & 11R-2 & 4 & 224 & Vla & 183,65 & 319,65 & $15 \mathrm{R}-3$ & 3 & 206. & MV & 266,35 & 60,35 & $20 \mathrm{R}-3$ & $2 \mathrm{C}$ & 248 & V2 & 220,27 & 332,27 \\
\hline $3 \mathrm{R}-2$ & 4 & 171 & V2 & 251,15 & 80,15 & $11 \mathrm{R}-3$ & 1 & 210 & Pf & 157,43 & 307,43 & $15 \mathrm{R}-3$ & 3 & 206. & V3 & 30,59 & 184,59 & $20 \mathrm{R}-3$ & $2 \mathrm{C}$ & 248 & V3 & 240,8 & 352,8 \\
\hline $3 \mathrm{R}-2$ & 4 & 171 & V3 & 81,50 & 270,50 & $11 \mathrm{R}-3$ & i & 210 & V2 & 157,43 & 307,43 & $15 R-4$ & 3 & 209 & $\mathrm{~V} 2$ & 160,70 & 311,70 & $20 \mathrm{R}-4$ & $3 \mathrm{~A}$ & 203 & v3 & 173,57 & 330.57 \\
\hline $4 \mathrm{R}-1$ & 5 & 223 & V3 & 75,35 & 212,35 & IIR-3 & 1 & 210 & $\mathrm{Pf}$ & 161,33 & 311,33 & $15 R-6$ & 5 & 295 & $\mathrm{J1}$ & 170,85 & 235,85 & $20 R-4$ & $3 \mathrm{~A}$ & 203 & Vla & 178,61 & 335,61 \\
\hline $4 \mathrm{R}-1$ & 5 & 223 & Af & 180,25 & 317,25 & IIR-3 & 4 & 192 & $\mathrm{Pf}$ & 149,39 & 317,39 & $15 \mathrm{R}-6$ & 5 & 295 & $\mathrm{~J} 2$ & 218,6 & 283,6 & $20 R-4$ & $3 \mathrm{~A}$ & 203 & V2 & 202,41 & 359,41 \\
\hline $4 \mathrm{R}-1$ & 5 & 223 & Pf & 180,25 & 317,25 & $11 \mathrm{R}-3$ & 4 & 192 & $\mathrm{v} 2$ & 149,39 & 317,39 & $15 \mathrm{R}-6$ & 5 & 295 & J3 & 245,26 & 310,26 & $20 \mathrm{R}-4$ & $3 \mathrm{~B}$ & 203 & $\mathrm{v} 2$ & 250,37 & 47,37 \\
\hline $4 \mathrm{R}-1$ & 5 & 223 & V2 & 180,25 & 317,25 & IIR-3 & 4 & 192 & $\mathrm{Pf}$ & 166,38 & 334,38 & $15 R-6$ & 5 & 295 & $\mathrm{~V} 2$ & 255,55 & 320,55 & $20 R-4$ & $3 \mathrm{~B}$ & 203 & Vla & 202,47 & 359,47 \\
\hline $4 \mathrm{R}-2$ & 3 & 214 & $\mathrm{~V} 2$ & 134,30 & 280,30 & $12 \mathrm{R}-1$ & 6 & 182 & $\mathrm{Pf}$ & 47,28 & 225,28 & $16 \mathrm{R}-1$ & 5 & 206 & $\mathrm{Pf}$ & 150,30 & 304,30 & $20 \mathrm{R}-5$ & 4 & 180 & $\mathrm{Pf}$ & 4,52 & 184,52 \\
\hline $4 \mathrm{R}-2$ & 3 & 214 & $\mathrm{~V} 2$ & 178,22 & 324,22 & $12 \mathrm{R}-2$ & 11 & 205 & V2 & 160,37 & 315,37 & $16 \mathrm{R}-2$ & 1 & 231 & $\mathrm{Pf}$ & 144,43 & 273,43 & 20R-5 & 4 & 180 & vi & 10,85 & 190,85 \\
\hline $4 \mathrm{R}-2$ & 6 & 318 & $\mathrm{v} 2$ & 235,31 & 277,31 & $12 \mathrm{R}-2$ & i1 & 205 & $\mathrm{Pf}$ & 178,28 & 333,28 & $16 \mathrm{R}-2$ & $i$ & 231 & V2 & 171,42 & 300,42 & $20 R-5$ & 4 & 180 & V2 & 29,65 & 209,65 \\
\hline $4 \mathrm{R}-3$ & 5 & 207 & V3 & 112,13 & 265,13 & $12 \mathrm{R}-3$ & 3 & 235 & V2 & 206,43 & 331,43 & 16R-3 & 1 & 250 & V1 & 359,69 & 109,69 & $21 R-2$ & 1 & 197 & vi & 328,68 & 131,68 \\
\hline $4 R-3$ & 5 & 207 & V2 & 142,37 & 295,37 & $12 \mathrm{R}-3$ & 4 & 246 & Pf & 191,39 & 305,39 & $16 R-4$ & IB & 185 & V2 & 189,29 & 4,29 & $21 R-3$ & i & 232 & $\mathrm{~V} 2$ & 345,85 & 113,85 \\
\hline $5 R-1$ & 10 & 29 & Af & 168,9 & 139,9 & $12 \mathrm{R}-3$ & 4 & 246 & V2 & 212,37 & 326,37 & $16 R-4$ & IB & 185 & Pf & 205,41 & 20,41 & $21 \mathrm{R}-3$ & 1 & 232 & Af & 135,15 & 263,15 \\
\hline $5 R-1$ & 10 & 29 & V2 & 171,72 & 142,72 & $12 R-4$ & 2 & 219 & Pf & 169,24 & 310,24 & $16 R-4$ & 1B & 185 & V1 & 296,60 & 111,60 & $2 \mathrm{IR}-3$ & 1 & 232 & Pf & 135,15 & 263,15 \\
\hline $5 \mathrm{R}-1$ & 10 & 29 & Pf & 172,32 & 143,32 & $12 \mathrm{R}-4$ & 2 & 219 & Vi & 178,82 & 319,82 & $16 \mathrm{R}-4$ & IB & 185 & V3 & 143,56 & 318,56 & $21 \mathrm{R}-3$ & i & 232 & v2 & 135,15 & 263,15 \\
\hline $5 \mathrm{R}-\mathrm{i}$ & 10 & 29 & v3 & 202,90 & 173,90 & $12 \mathrm{R}-4$ & 4 & 234 & Pf & 165,23 & 291,23 & $16 \mathrm{R}-4$ & IC & 185 & Pf & 205,41 & 20,41 & $2 \mathrm{IR}-3$ & i & 232 & VIb & 158,52 & 86,5 \\
\hline $5 \mathrm{R}-2$ & 1 & 188 & V $3 b$ & 229,85 & 41,85 & $12 R-4$ & 4 & 234 & V2 & 195,25 & 321,25 & $16 R-4$ & IC & 185 & V2 & 212,43 & 27,43 & $21 R-3$ & i & 232 & Vla & 172,80 & 300,80 \\
\hline $5 \mathrm{R}-2$ & 1 & 188 & V2 & 154,27 & 326,27 & $12 \mathrm{R}-5$ & 3 & 120 & V2 & 105,56 & 345,56 & $16 R-4$ & IC & 185 & V1 & 235,75 & 50,75 & $2 \mid \mathrm{R}-4$ & 6 & 222 & Vla & 184,28 & 322,28 \\
\hline $5 \mathrm{R}-2$ & i & 188 & $\mathrm{Pf}$ & 175,23 & 347,23 & $12 \mathrm{R}-5$ & 7 & 246 & $\mathrm{Pf}$ & 146,43 & 260,43 & $16 R-5$ & 1 & 253 & $\mathrm{Pf}$ & 163,23 & 270 . & $21 R-4$ & 6 & 222 & Af & 195,26 & 333,26 \\
\hline $5 \mathrm{R}-2$ & 1 & $\begin{array}{l}100 \\
188\end{array}$ & V3a & 175,64 & 347,64 & $12 \mathrm{R}-5$ & 7 & 246 & Af & 180,32 & 294,32 & $16 \mathrm{R}-5$ & 1 & 253 & V2 & 167,29 & 274,29 & $2 \mathrm{IR}-4$ & 6 & 222 & $\mathrm{Pf}$ & 195,26 & 333,26 \\
\hline $5 \mathrm{R}-3$ & 2 & 265 & $\mathrm{~V} 1$ & 147,21 & 242,21 & $12 \mathrm{R}-5$ & 7 & 246 & $\mathrm{~V} 2$ & 180,32 & 294,32 & $16 \mathrm{R}-5$ & 4 & 224 & $\mathrm{v} 2$ & 161,21 & 297,21 & $21 R-4$ & 6 & 222 & V2 & 195,26 & 333,26 \\
\hline $5 R-3$ & 13 & 206 & $\mathrm{Pf}$ & 206,34 & 0,34 & $13 R-2$ & 3 & 270 & Af & 180,30 & 270,30 & $16 \mathrm{R}-5$ & 4 & 224 & vo & 0,75 & 136,75 & $22 \mathrm{R}-2$ & 1 & 249 & Af & 264,9 & 15,9 \\
\hline $5 R-3$ & 13 & 206 & V1 & 206,34 & 0,34 & $13 \mathrm{R}-2$ & 3 & 270 & $\mathrm{Pf}$ & 180,30 & 270,30 & $16 \mathrm{R}-7$ & 6 & 278 & $\mathrm{~V} 2$ & 145,12 & 227,12 & $22 \mathrm{R}-2$ & 1 & 249 & $\mathrm{~V} 2$ & 264,9 & 15,9 \\
\hline $5 R-3$ & 13 & 206 & $\mathrm{Pf}$ & 236,41 & 30,41 & $13 \mathrm{R}-2$ & 3 & 270 & V1 & 180,30 & 270,30 & $16 R-7$ & 6 & 278 & Pf & 214,11 & 296,11 & $22 \mathrm{R}-2$ & i & 249 & $\mathrm{Pf}$ & 163,10 & 274,10 \\
\hline $5 R-4$ & 6 & 199 & V2 & 184,29 & 345,29 & $13 \mathrm{R}-2$ & 3 & 270 & $\mathrm{~V} 2$ & 180,30 & 270,30 & $16 \mathrm{R}-7$ & 6 & 278 & MV & 260,40 & 342 & $22 \mathrm{R}-2$ & 1B & 249 & Vib & 293,83 & 44,83 \\
\hline $5 R-4$ & 7 & 200 & V2 & 169,49 & 329,49 & $13 R-3$ & 19 & 176 & v0 & 194,84 & 18,84 & 17R-1 & 1 & 347 & MV & 142,22 & 155 & $22 \mathrm{R}-2$ & IC & 24 & Pf & 173,17 & 284,17 \\
\hline $6 \mathrm{R}-1$ & 6 & 218 & $\mathrm{Pf}$ & 165,46 & 307,46 & $13 R-3$ & 19 & 176 & Af & 221,23 & 45,23 & $17 \mathrm{R}-1$ & i & 347 & V2 & 172,32 & 185 & $22 \mathrm{R}-2$ & IC & 24 & V2 & 173,17 & 284,17 \\
\hline $6 \mathrm{R}-1$ & 6 & 218 & $\mathrm{~V} 2$ & 170,49 & 312,49 & $13 \mathrm{R}-3$ & 19 & 176 & $\mathrm{Pf}$ & 221,23 & 45,23 & $17 \mathrm{R}-3$ & i & 222 & Pf & 235,45 & 13 , & $22 \mathrm{R}-2$ & ID & 24 & Pf & 170,16 & 281,16 \\
\hline $6 \mathrm{R}-2$ & 9 & 273 & $\mathrm{~V}_{1}$ & 239,12 & 326 , & $13 R-3$ & 19 & 176 & $\mathrm{Vb}$ & 221,23 & 45,2 & $17 \mathrm{R}-3$ & 1 & 222 & V2 & 235,45 & 13, & $22 \mathrm{R}-3$ & $1 \mathrm{~A}$ & 21 & Vla & 178,65 & 322,65 \\
\hline $6 \mathrm{R}-3$ & 7 & 200 & V2 & 45,61 & 205,61 & $13 R-3$ & 19 & 176 & V3 & 18,87 & 202,87 & $17 R-3$ & 1 & 222 & vo & 120,63 & 258,63 & $22 \mathrm{R}-3$ & 1B & 21 & V2 & 299,10 & 83,10 \\
\hline $6 \mathrm{R}-3$ & 7 & 200 & $\mathrm{Pf}$ & 48,60 & 208,60 & $13 R-4$ & 6 & 188 & Vla & 323,61 & 135,61 & $17 \mathrm{R}-4$ & 3 & 229 & Af & 225,39 & 356 . & $22 \mathrm{R}-3$ & IB & 21 & $\mathrm{CL}$ & 166,83 & 10,8 \\
\hline $6 \mathrm{R}-3$ & 7 & 200 & V1 & 96,41 & 256,41 & $13 R-4$ & 6 & 188 & Af & 170,30 & 342,30 & $17 \mathrm{R}-4$ & 3 & 229 & $\mathrm{Pf}$ & 225,39 & 356 , & $22 R-3$ & IB & 21 & $\mathrm{~V} 3$ & 202,79 & 346,79 \\
\hline $7 \mathrm{R}-1$ & 17 & 198 & V1a & 139,11 & 301,11 & $13 R-4$ & 6 & 188 & $\mathrm{Pf}$ & 170,30 & 342,30 & $17 R-4$ & 3 & 229 & v2 & 225,39 & 356,3 & $22 \mathrm{R}-4$ & 1 & 170 & Pf & 127,46 & 317,46 \\
\hline $8 \mathrm{R}-2$ & 3 & 167 & 1 & 200,47 & 33,47 & 13R-4 & 6 & 188 & V1b & 170,30 & 342,30 & $17 \mathrm{R}-4$ & 3 & 229 & $\mathrm{~V} 3$ & 225,39 & 356,39 & $22 R-4$ & IA & 170 & V2 & 166,41 & 356,41 \\
\hline $10 \mathrm{R}-2$ & 7 & 190 & MV & 205,72 & 15,72 & $14 \mathrm{R}-3$ & 3 & 110 & V1 & 21,16 & 271,16 & $18 \mathrm{R}-2$ & 9 & 295 & $\mathrm{~V} 2 \mathrm{a}$ & 5,82 & 70,8 & $22 R-5$ & 6 & 203 & $\mathrm{v} 2$ & 5,89 & 162,89 \\
\hline $10 \mathrm{R}-2$ & 7 & 190 & Vla & 172,50 & 342,50 & $14 R-3$ & 4 & 338 & $\mathrm{~V} 2$ & 351,30 & 13,3 & $18 \mathrm{R}-2$ & 9 & 295 & Pf & 248,37 & 313,37 & $22 \mathrm{R}-5$ & 6 & 203 & $\mathrm{v}_{1}$ & 10,85 & 167,85 \\
\hline $10 \mathrm{R}-2$ & 7 & 190 & $\mathrm{~V} 2$ & 0,80 & 350 , & & 4 & 33 & Vib & 172,43 & 194. & & 9 & 295 & vo & 248,37 & 313,3 & $22 \mathrm{R}-5$ & 6 & 203 & $\mathrm{~V} 3 \mathrm{a}$ & 186,78 & 343,78 \\
\hline $10 \mathrm{~F}$ & 9 & 202 & $\mathrm{~V} 3$ & & & & 4 & & V3a & & 22,4 & & 3 & 213 & V1 & & & & 2 & 22 & V2 & 227,87 & $4,87^{\circ}$ \\
\hline $10 R$ & 9 & 20 & Af & & & & 8 & 16 & V1 & & & & 3 & 21 & $\mathrm{Pf}$ & & & & 2 & 22 & MV & & 116,44 \\
\hline $10 \mathrm{R}-3$ & 9 & 202 & Pf & & & & 5 & 212 & Af & & & $19 \mathrm{R}$ & 3 & 213 & V2 & 147 & 294 & & 2 & 22 & V2 & 340,12 & 117,12 \\
\hline 10R-3 & 9 & 202 & V2 & & 342,3 & $14 R$ & 5 & 212 & Pf & 175,24 & 323,24 & $19 \mathrm{R}$ & 1 & 195 & V1 & 242,61 & 47,61 & 221 & 8 & 180 & V0 & 180,11 & 0,11 \\
\hline $10 \mathrm{R}-4$ & 7 & 233 & vo & 80,30 & 207,30 & $14 \mathrm{R}-5$ & 5 & 212 & V2 & 175,24 & 323,24 & $20 \mathrm{R}-1$ & 13 & 197 & vi & 18,58 & 181,58 & $22 \mathrm{R}-7$ & 8 & 180 & MV & 357.79 & 177,79 \\
\hline $10 \mathrm{R}-4$ & 7 & 233 & Pf & 185,33 & 312,33 & $14 \mathrm{R}-5$ & 57 & 52 & $\mathrm{~V} 2 \mathrm{~b}$ & 152,17 & 100,17 & $20 \mathrm{R}-1$ & 13 & 197 & vo & 156,31 & 319,31 & $22 \mathrm{R}-7$ & 8 & 180 & V2 & 117,40 & 297,40 \\
\hline $10 \mathrm{R}-4$ & 7 & 233 & V3 & 208,64 & 335,64 & & & & & & & & & & & & & & & & & & \\
\hline
\end{tabular}

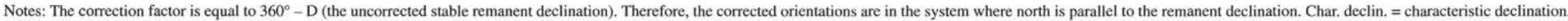
uncorr. $=$ uncorrected, corr. = corrected. Feature abbreviations are described in Shipboard Scientific Party (1995a) and in other portions of this volume (Dilek, Coulton, et al., this volume). 
Top of Archive half of core

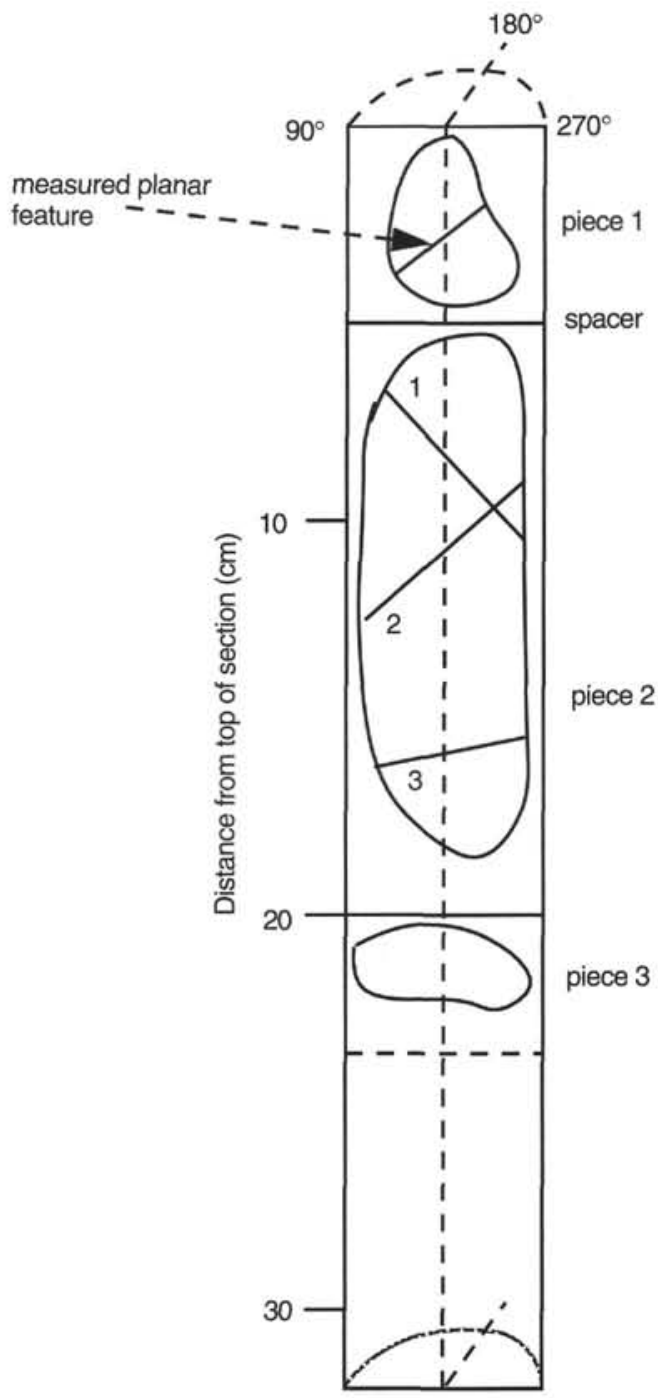

Figure 3. Sketch of the coordinate system for measuring features in ODP core. Minicores for paleomagnetic analysis were drilled from the working half of the core with the axis of the minicore cylinder aligned along the $0^{\circ}$ direction. Measured declinations were converted to this core reference frame and then used to reorient structural features on a piece-by-piece basis.

the declination. For example, a $14^{\circ}$ counterclockwise rotation about a $340^{\circ}$ axis would change a $41^{\circ}$ inclination to $35^{\circ}$, and at the same time change the declination from $0^{\circ}$ to $10^{\circ}$. Therefore, if the inclination discrepancy is due entirely to rotations about north-south-trending axes, and the characteristic declination is assumed to be defined by a geocentric axial dipole field, then returning the characteristic declination to $0^{\circ}$ will add a significant error to the reorientation of the core.

Despite the significant uncertainties associated with the unknown effects of anisotropy and tectonic rotation on the remanent declination, we assumed that the observed declination for each discrete sample was originally $0^{\circ}$. Reorientation of these samples (Site 920) involves subtracting the measured declination from any azimuth measurement done in the ODP core reference frame (Fig. 3). Stereographic projections of the reoriented major fabric in core from Site 920 show there to be a strong clustering of the poles to the crystal-plastic and V2 vein set, which comprise a planar fabric. This clustering indicates that this mesoscopic fabric has a consistent orientation downhole and between holes.

\section{RESULTS}

After reorientation, the mesoscopic crystal-plastic foliation and V2 vein set are strongly clustered with north-northwest strikes and east-northeast dips. The east-northeast dip of the foliation is parallel to in situ observations of a generally east-dipping fabric in nearby serpentinite outcrop by submersibles and towed cameras (Karson and Dick, 1983; Cannat et al., 1995; J. Miller, unpubl. data). Paleomagnetic measurements on two oriented samples collected at Site 920 during an Alvin dive in the spring of 1995 produced disparate results (Fig. 6A). However, one sample had a measured declination of $336^{\circ}$ and an inclination of $42^{\circ}$; the direction is similar to the present-day International Geomagnetic Reference Field at the site. The submersible samples show similar fabric elements and magnetic properties as samples from Site 920 core. The discrepancy in results from the two submersible samples is more likely to be from misorientation of the sample upon recovery after the Alvin dive. Attempts to collect oriented samples during Alvin dives usually result in high failure rates because of ambiguity about which sample surfaces were measured and undulating rock surfaces that make reorientation subject to large errors.

There is no significant difference in the distribution of reoriented planar structures when data from each hole are analyzed separately. Magmatic veins (MV) and an early generation of mineralized veins (V0; Fig. 6C) generally scatter with no preferred orientation. These early veins consist usually of coarse-grained mafic minerals that are considered to be late-stage melt segregations (Cannat, Ceuleneer, et al., this volume), and that tend to cut the core at random orientations (Shipboard Scientific Party, 1995b). Two other vein generations, an early set (V1) that is cut by V2 veins and a vein set (V3) that typically cuts the V2 veins at a steep angle (Fig. 6D), are observed in the core. In general, there is no clustering of the orientations of the $\mathrm{V} 1$ vein set; however, the V3 set has a subtle clustering that results in dips to the northeast with very few, if any, strike orientations in that quadrant. Some of the scatter in plots incorporating data from different vein sets may reflect the misinterpretation and misassignment of the measured vein to a specific set. Not all the vein sets were present along all intervals of core. Particularly, if the V2 vein set was not well developed, then the most common crosscutting relationships could not be well established and classification was purely on the basis of the appearance of the vein on the cut surface of the core.

In Hole 920B, the plastic fabric is nearly always parallel to the orientation of the V2 vein set. The strike of V2 veins are consistently north-northwest, although in the middle of shipboard Unit 3 (35-85 mbsf), there is a cluster that strikes north-northeast (Fig. 7). Coincident with this cluster is an increase in the dip of the V2 veins. In general, the V3 veins show steeper dips, except near the same location downhole in Unit 3, where some relatively shallow dips were observed.

V2 veins in core from Hole 920D (Fig. 8) show more variation in both strike and dip than those in core from Hole 920B. In the upper part of Hole 920D, the vein orientations are similar to that in Hole $920 \mathrm{~B}$, and the crystal-plastic fabric is generally parallel to the V2 veins. Below $110 \mathrm{~m}$ depth, the strike and dip of both V2 and the plastic fabric vary more strongly and substantially diverge from each other. The development of $\mathrm{V} 2$ veins is sparse in many intervals of core below this depth. The interpretation of these patterns and the significance of the vein generations at Site 920 is discussed in Dilek, Coulton, et al. (this volume). Measurements of faults, joints, lineations, and structural elements other than plastic fabric and veins are too sparse for any general interpretations to be made; however, their orientations are included in Tables 1 and 2.

Structural features in the gabbro cores are much less common and more difficult to measure accurately, partly because much of the magmatic and plastic deformation pervasive in these cores is weakly developed (Shipboard Scientific Party, 1995c, 1995d). The magnetic behavior of the samples taken from gabbroic rocks is also much more complicated (Gee et al., this volume). Some gabbro samples from 
Table 3. Anisotrophy of magnetic susceptibility (AMS) data for Holes 920B and 920D.

\begin{tabular}{|c|c|c|c|c|c|c|c|c|c|c|c|}
\hline \multirow[b]{2}{*}{$\begin{array}{l}\text { Core, section, } \\
\text { interval }(\mathrm{cm})\end{array}$} & \multirow[b]{2}{*}{$\begin{array}{l}\text { Depth } \\
\text { (mbsf) }\end{array}$} & \multicolumn{3}{|c|}{ AMS } & \multirow[b]{2}{*}{$\begin{array}{l}\text { Char. declin. } \\
\text { (uncorr.) }\end{array}$} & \multicolumn{6}{|c|}{ AMS directions, reoriented } \\
\hline & & $K_{1} / K_{3}$ & $K_{1} / K_{2}$ & $K_{2} / K_{3}$ & & $\begin{array}{c}K_{l} \\
\text { declin. }\end{array}$ & $\begin{array}{c}K_{l} \\
\text { inclin. }\end{array}$ & $\begin{array}{c}K_{2} \\
\text { declin. }\end{array}$ & $\begin{array}{c}K_{2} \\
\text { inclin. }\end{array}$ & $\begin{array}{c}K_{3} \\
\text { declin. }\end{array}$ & $\begin{array}{c}K_{3} \\
\text { inclin. }\end{array}$ \\
\hline \multicolumn{12}{|l|}{ 153-920B- } \\
\hline $1 \mathrm{~W}-1,55-58$ & 0.55 & 1.2030 & 1.0780 & 1.1160 & 244.0 & 123.4 & 51.6 & $7.7^{\circ}$ & 18.9 & 265.3 & 31.9 \\
\hline $1 \mathrm{~W}-2,24-27$ & 1.29 & 1.1140 & 1.0170 & 1.0950 & 181.0 & 21.5 & 28.7 & 125.8 & 24.2 & 249.3 & 50.8 \\
\hline $1 \mathrm{~W}-3,46-49$ & 2.94 & 1.1110 & 1.0450 & 1.0640 & 187.5 & 21.1 & 17.5 & 119.2 & 23.9 & 258.3 & 59.7 \\
\hline IW $-3,73-76$ & 3.21 & 1.1583 & 1.0499 & 1.1032 & 34.0 & 46.9 & 27.3 & & & 286.1 & 44.7 \\
\hline $1 \mathrm{~W}-3,76-79$ & 3.24 & 1.1716 & 1.0641 & 1.1010 & 54.0 & 50.0 & 26.1 & & & 262.8 & 59.8 \\
\hline $2 \mathrm{R}-1,17-20$ & 14.17 & 1.1090 & 1.0350 & 1.0710 & 294.2 & 359.1 & 3.0 & 89.7 & 11.7 & 255.1 & 77.8 \\
\hline $3 R-1,65-68$ & 24.15 & 1.2190 & 1.0560 & 1.1540 & 193.1 & 5.4 & 9.3 & 107.5 & 52.2 & 268.6 & 36.2 \\
\hline $3 R-2,36-39$ & 25.36 & 1.1200 & 1.0730 & 1.0440 & 163.6 & 0.9 & 7.8 & 99.2 & 46.2 & 263.7 & 42.7 \\
\hline $3 R-2,79-83$ & 25.79 & 1.1510 & 1.1048 & 1.0415 & 194.0 & 211.5 & 12.9 & & & 117.6 & 16.2 \\
\hline $3 R-2,91-94$ & 25.91 & 1.2114 & 1.0946 & 1.1670 & 194.0 & 212.2 & 24.9 & 315.5 & 58.5 & 113.6 & 18.0 \\
\hline $3 R-2,98-101$ & 25.98 & 1.2130 & 1.0850 & 1.1170 & 196.5 & 15.1 & 19.0 & 141.7 & 60.0 & 277.1 & 22.3 \\
\hline $4 \mathrm{R}-1,60-63$ & 33.80 & 1.1070 & 1.0500 & 1.0540 & 266.5 & 16.8 & 27.3 & 146.6 & 51.2 & 272.6 & 25.3 \\
\hline $5 \mathrm{R}-1,52-55$ & 43.22 & 1.1700 & 1.1110 & 1.0530 & 217.5 & 3.91 & 5.81 & 50.97 & 1.42 & 71.1 & 9.6 \\
\hline $5 R-2,60-63$ & 44.74 & 1.1880 & 1.0460 & 1.1350 & 204.8 & 21.0 & 27.0 & 130.5 & 33.2 & 260.8 & 44.6 \\
\hline $5 R-3,61-64$ & 46.23 & 1.0880 & 1.0580 & 1.0290 & 207.2 & 6.6 & 13.3 & 250.4 & 61.8 & 102.8 & 24.4 \\
\hline $5 R-3,99-102$ & 46.61 & 1.1890 & 1.0940 & 1.0870 & 182.7 & 4.2 & 6.4 & 105.6 & 60.5 & 270.7 & 28.7 \\
\hline $6 \mathrm{R}-3,21-23$ & 55.05 & 1.0890 & 1.0490 & 1.0380 & 228.2 & 11.2 & 43.4 & 188.4 & 46.6 & 279.9 & 1.4 \\
\hline $6 \mathrm{R}-3,23-26$ & 55.08 & 1.0730 & 1.0240 & 1.0470 & 239.1 & 22.0 & 40.2 & 127.1 & 17.2 & 235.1 & 44.8 \\
\hline $6 \mathrm{R}-3,57-60$ & 55.42 & 1.0640 & 1.0540 & 1.0100 & 222.1 & 187.6 & 24.6 & 46.5 & 59.5 & 285.6 & 16.8 \\
\hline $7 \mathrm{R}-1,20-23$ & 61.20 & 1.1307 & 1.0451 & 1.0819 & 232.0 & 258.8 & 26.9 & 36.9 & 55.8 & 158.4 & 19.6 \\
\hline $7 \mathrm{R}-1,23-26$ & 61.23 & 1.0957 & 1.0395 & 1.0541 & 217.0 & 253.3 & 20.1 & 7.2 & 47.9 & 148.3 & 35.2 \\
\hline $7 \mathrm{R}-1,55-58$ & 61.55 & 1.1980 & 1.1200 & 1.0700 & 209.5 & 15.11 & 1.31 & 40.8 & 71.1 & 282.0 & 14.9 \\
\hline $7 R-2,5-8$ & 62.47 & 1.2660 & 1.0280 & 1.2310 & 176.2 & 358.9 & 0.28 & 9.15 & 3.52 & 68.83 & 6.5 \\
\hline $7 R-2,42-45$ & 62.84 & 1.2390 & 1.0970 & 1.1300 & 194.7 & 169.2 & 0.97 & 8.24 & 8.52 & 60.04 & 1.5 \\
\hline $7 R-2,91-94$ & 63.33 & 1.1860 & 1.0480 & 1.1320 & 178.3 & 174.9 & 0.7 & 84.0 & 53.4 & 265.5 & 36.5 \\
\hline $7 \mathrm{R}-3,15-18$ & 63.98 & 1.1580 & 1.0520 & 1.1000 & 193.1 & 157.2 & 10.5 & 55.9 & 46.5 & 256.6 & 41.5 \\
\hline $8 \mathrm{R}-1,81-84$ & 71.21 & 1.1734 & 1.0474 & 1.1202 & 181.0 & 182.0 & 2.4 & 279.0 & 71.1 & 91.2 & 18.7 \\
\hline $8 \mathrm{R}-1,84-87$ & 71.24 & 1.2089 & 1.0605 & 1.1400 & 183.0 & 356.9 & 14.2 & 235.4 & 64.1 & 92.5 & 21.2 \\
\hline $8 R-2,24-27$ & 71.92 & 1.2540 & 1.0490 & 1.1960 & 195.0 & 167.9 & 3.1 & 75.1 & 42.5 & 261.2 & 47.4 \\
\hline $8 R-2,70-73$ & 72.38 & 1.1260 & 1.0650 & 1.0570 & 187.0 & 197.7 & 4.3 & 292.9 & 49.7 & 104.1 & 39.9 \\
\hline $8 R-2,73-76$ & 72.41 & 1.0740 & 1.0410 & 1.0320 & 187.0 & 189.9 & 12.3 & 63.5 & 69.8 & 283.4 & 15.7 \\
\hline $8 R-3,69-72$ & 73.71 & 1.1700 & 1.0560 & 1.1080 & 190.2 & 1.7 & 0.2 & 91.9 & 53.4 & 271.6 & 36.7 \\
\hline $8 \mathrm{R}-3,115-118$ & 74.17 & 1.1560 & 1.1000 & 1.0500 & 214.8 & 10.2 & 29.4 & 133.3 & 44.1 & 260.0 & 31.6 \\
\hline $8 \mathrm{R}-3,118-121$ & 74.20 & 1.1520 & 1.0600 & 1.0870 & 213.8 & 10.6 & 32.7 & 155.2 & 51.8 & 268.9 & 17.6 \\
\hline $8 R-4,11-14$ & 74.48 & 1.1230 & 1.0180 & 1.1040 & 189.0 & 25.9 & 26.9 & 178.0 & 60.2 & 289.7 & 11.9 \\
\hline $8 R-4,17-20$ & 74.54 & 1.1408 & 1.0020 & 1.1385 & 180.0 & 201.8 & 24.3 & 328.2 & 52.7 & 98.92 & 6.4 \\
\hline $8 R-4,29-32$ & 74.66 & 1.1351 & 1.0129 & 1.1206 & 191.0 & 219.0 & 66.2 & 14.9 & 21.9 & 108.5 & 8.8 \\
\hline $8 R-5,62-65$ & 76.36 & 1.1920 & 1.0590 & 1.1250 & 185.2 & 9.2 & 10.8 & 113.8 & 53.1 & 271.5 & 34.8 \\
\hline $9 \mathrm{R}-1,41-44$ & 80.11 & 1.1380 & 1.1070 & 1.0280 & 200.4 & 8.3 & 14.4 & 149.3 & 71.7 & 275.4 & 11.0 \\
\hline $9 R-2,36-39$ & 81.52 & 1.1110 & 1.0460 & 1.0620 & 236.7 & 14.8 & 24.4 & 130.9 & 44.1 & 265.6 & 36.0 \\
\hline $9 \mathrm{R}-2,39-42$ & 81.55 & 1.1040 & 1.0500 & 1.0510 & 236.7 & 17.2 & 38.9 & 133.1 & 28.5 & 248.2 & 38.0 \\
\hline $9 \mathrm{R}-2,69-72$ & 81.85 & 1.1144 & 1.0439 & 1.0676 & 202.0 & 25.1 & 54.2 & 190.9 & 35.0 & 285.6 & 6.8 \\
\hline $9 \mathrm{R}-2,72-75$ & 81.88 & 1.1106 & 1.0360 & 1.0721 & 212.0 & 212.4 & 59.4 & 13.9 & 29.3 & 108.5 & 8.1 \\
\hline $9 \mathrm{R}-2,84-87$ & 82.00 & 1.1480 & 1.0070 & 1.1400 & 194.7 & 353.1 & 65.6 & 172.3 & 24.3 & 262.4 & 0.3 \\
\hline $9 \mathrm{R}-2,87-90$ & 82.03 & 1.1390 & 1.0220 & 1.1140 & 194.9 & 349.3 & 50.9 & 153.2 & 38.0 & 249.4 & 8.0 \\
\hline $10 \mathrm{R}-1,17-20$ & 89.27 & 1.2010 & 1.0480 & 1.1460 & 216.8 & 339.8 & 1.3 & 71.6 & 55.9 & 249.0 & 34.1 \\
\hline $10 \mathrm{R}-1,20-23$ & 89.30 & 1.2120 & 1.0050 & 1.2060 & 216.8 & 156.8 & 15.4 & 37.3 & 60.7 & 254.0 & 24.3 \\
\hline $10 \mathrm{R}-2,2-5$ & 90.48 & 1.0690 & 1.0320 & 1.0360 & 192.5 & 180.5 & 3.0 & 83.5 & 66.5 & 271.7 & 23.3 \\
\hline $10 \mathrm{R}-2,37-40$ & 90.83 & 1.1154 & 1.0295 & 1.0835 & 220.0 & 249.3 & 46.6 & 0.5 & 18.9 & 105.5 & 37.3 \\
\hline $10 \mathrm{R}-2,63-66$ & 91.09 & 1.3030 & 1.0247 & 1.2716 & 200.0 & 228.8 & 52.9 & 351.9 & 22.4 & 94.5 & 27.8 \\
\hline $10 \mathrm{R}-2,70-73$ & 91.16 & 1.2960 & 1.0630 & 1.2200 & 207.7 & 353.4 & 16.3 & 111.8 & 58.5 & 255.1 & 26.1 \\
\hline
\end{tabular}

Sites 921 to 924 do have a relatively simple demagnetization behavior, where a single characteristic component of magnetization can be identified with an inclination near the expected value of $41^{\circ}$. Veins from Hole $923 \mathrm{~A}$ have been reoriented using the same method as for Site 920 and using only data where this characteristic inclination is near $41^{\circ}$ (Fig. 9). Although the number of structures including both magmatic fabrics and metamorphic foliations as well as veins is low, the bulk of them have a gentle dip to the northeast after reorientation (Table 4). Again, this is consistent with structural evidence from dive programs, and is an indication that, with further work on gabbro samples to isolate characteristic magnetization components, it should be possible to reorient many more features in core from Holes 921A to 924C.

\section{CONCLUSIONS}

The reorientation of planar features in Site 920 cores using a characteristic remanent declination of the magnetization shows that major pervasive structural fabric elements in the core have a consistent orientation that dips to the east-northeast. Submersible studies have shown that surface outcrops of the serpentinites commonly have a gently east-dipping structure defined by a disjunctive, anastomosing foliation similar to that observed in the core from Site 920. This con- sistency indicates that the reorientation procedure is reasonably valid for these rocks.

The V2 veins and crystal-plastic fabric reflect deformation in at least two different temperature and pressure regimes. However, only serpentinization and possible later magnetization events, including the creation of the V2 serpentine vein set, affected the acquired remanence. A high anisotropy of susceptibility is observed in these samples, probably caused by preferred orientation of magnetite grains or grain clusters formed during the serpentinization (Lawrence et al., this volume). This anisotropy may account for part of the discrepancy between measured inclinations $(35.2 \pm 1)$ and the expected geocentric axial dipole inclination $\left(41^{\circ}\right)$ for the site.

Although the major fabric elements generally show consistent orientations throughout the core, other less conspicuous structural features show no obvious relationship to the major foliations or each other. Vein sets other than the V2 set generally have randomly scattered orientations overall, with no discernible relationship to depth in the recovered core or rock type.

There is generally a more diverse set of structural elements in the gabbros recovered from Sites 921 to 924 . Preliminary reorientation of some of these elements in core from Hole 923A indicates an eastnortheast dip of many of these structures, which is consistent with the regional trends and local submersible observations of gabbro exposures. 
Table 3 (continued).

\begin{tabular}{|c|c|c|c|c|c|c|c|c|c|c|c|}
\hline \multirow[b]{2}{*}{$\begin{array}{l}\text { Core, section, } \\
\text { interval }(\mathrm{cm})\end{array}$} & \multirow[b]{2}{*}{$\begin{array}{l}\text { Depth } \\
\text { (mbsf) }\end{array}$} & \multicolumn{3}{|c|}{ AMS } & \multirow[b]{2}{*}{$\begin{array}{l}\text { Char. declin. } \\
\text { (uncorr.) }\end{array}$} & \multicolumn{6}{|c|}{ AMS directions, reoriented } \\
\hline & & $K_{i} / K_{3}$ & $K_{1} / K_{2}$ & $K_{2} / K_{3}$ & & $\begin{array}{c}K_{l} \\
\text { declin. }\end{array}$ & $\begin{array}{c}K_{l} \\
\text { inclin. }\end{array}$ & $\begin{array}{c}K_{2} \\
\text { declin. }\end{array}$ & $\begin{array}{c}K_{2} \\
\text { inclin. }\end{array}$ & $\begin{array}{c}K_{3} \\
\text { declin. }\end{array}$ & $\begin{array}{c}K_{3} \\
\text { inclin. }\end{array}$ \\
\hline $10 \mathrm{R}-3,120-123$ & 93.07 & 1.1640 & 1.0240 & 1.1370 & 189.1 & 177.8 & 0.5 & 80.9 & 86.6 & 267.8 & 3.4 \\
\hline $10 \mathrm{R}-4,64-67$ & 93.95 & 1.1820 & 1.0320 & 1.1450 & 222.9 & 175.0 & 16.4 & 49.9 & 62.8 & 271.5 & 21.0 \\
\hline $10 \mathrm{R}-5,13-16$ & 94.87 & 1.1130 & 1.0440 & 1.0660 & 208.3 & 170.7 & 38.5 & 39.8 & 39.5 & 284.7 & 27.1 \\
\hline $11 \mathrm{R}-1,15-18$ & 98.65 & 1.1343 & 1.0284 & 1.1030 & 222.0 & 264.0 & 36.1 & 173.8 & 0.2 & 83.5 & 53.9 \\
\hline $11 R-1,57-60$ & 99.07 & 1.3010 & 1.0429 & 1.2475 & 212.0 & 247.0 & 36.5 & 359.4 & 27.3 & 116.4 & 41.4 \\
\hline $11 \mathrm{R}-1,70-73$ & 99.20 & 1.2690 & 1.0500 & 1.2080 & 206.2 & 7.8 & 17.4 & 113.3 & 40.4 & 259.9 & 44.6 \\
\hline $11 \mathrm{R}-1,119-122$ & 99.69 & 1.2320 & 1.0260 & 1.2010 & 250.8 & 5.1 & 12.0 & 119.9 & 63.0 & 269.7 & 23.8 \\
\hline $11 \mathrm{R}-1,122-125$ & 99.72 & 1.2260 & 1.0290 & 1.1910 & 250.8 & 9.6 & 24.4 & 132.6 & 50.2 & 264.9 & 29.3 \\
\hline $11 \mathrm{R}-2,10-13$ & 100.06 & 1.2860 & 1.0530 & 1.2210 & 206.0 & 1.6 & 10.2 & 102.4 & 46.1 & 262.3 & 42.1 \\
\hline $12 \mathrm{R}-1,51-54$ & 108.21 & 1.1680 & 1.0210 & 1.1430 & 245.8 & 62.7 & 46.7 & 168.4 & 14.3 & 270.7 & 39.8 \\
\hline $12 \mathrm{R}-1,66-69$ & 108.36 & 1.1746 & 1.0785 & 1.0891 & 246.0 & 253.0 & 22.1 & 16.3 & 53.5 & 150.8 & 27.4 \\
\hline $12 \mathrm{R}-1,76-79$ & 108.46 & 1.1566 & 1.0553 & 1.0959 & 246.0 & 270.9 & 15.1 & 20.9 & 51.7 & 170.4 & 34.2 \\
\hline $12 \mathrm{R}-1,100-103$ & 108.70 & 1.1810 & 1.0510 & 1.1240 & 183.1 & 106.0 & 40.7 & 325.3 & 42.0 & 215.2 & 20.9 \\
\hline $12 \mathrm{R}-2,50-53$ & 109.65 & 1.1800 & 1.0250 & 1.1510 & 193.6 & 132.7 & 47.3 & 7.1 & 28.2 & 259.8 & 29.1 \\
\hline $12 \mathrm{R}-2,113-116$ & 110.28 & 1.2376 & 1.0437 & 1.1858 & 193.0 & 5.9 & 13.8 & 260.6 & 47.0 & 107.6 & 39.7 \\
\hline $12 \mathrm{R}-2,117-120$ & 110.32 & 1.1961 & 1.0351 & 1.1555 & 192.0 & 11.5 & 0.4 & 280.3 & 70.7 & 101.6 & 19.3 \\
\hline $12 \mathrm{R}-2,126-129$ & 110.41 & 1.2276 & 1.0476 & 1.1718 & 244.0 & 50.7 & 23.6 & 278.5 & 57.0 & 150.7 & 21.7 \\
\hline $12 \mathrm{R}-2,129-132$ & 110.44 & 1.1513 & 1.0418 & 1.1051 & 251.0 & 49.1 & 32.2 & 267.4 & 51.2 & 151.7 & 19.2 \\
\hline $12 \mathrm{R}-3,26-29$ & 110.91 & 1.2468 & 1.0144 & 1.2292 & 202.0 & 2.1 & 36.1 & 228.4 & 43.5 & 112.1 & 25.1 \\
\hline $12 \mathrm{R}-3,29-32$ & 110.94 & 1.3040 & 1.0370 & 1.2574 & 193.0 & 197.0 & 9.5 & 325.3 & 74.9 & 105.0 & 11.6 \\
\hline $12 \mathrm{R}-3,80-83$ & 111.45 & 1.2640 & 1.0370 & 1.2180 & 224.0 & 9.3 & 21.4 & 122.8 & 45.4 & 262.2 & 36.9 \\
\hline $12 \mathrm{R}-4,80-83$ & 112.52 & 1.2160 & 1.0250 & 1.1860 & 197.0 & 332.4 & 1.2 & 63.4 & 37.8 & 240.9 & 52.2 \\
\hline $12 \mathrm{R}-5,10-13$ & 113.10 & 1.2410 & 1.0470 & 1.1850 & 214.3 & 30.6 & 16.7 & 146.9 & 56.0 & 291.1 & 28.7 \\
\hline $12 \mathrm{R}-5,13-16$ & 113.13 & 1.1940 & 1.0780 & 1.1070 & 214.3 & 87.9 & 43.1 & 309.7 & 38.5 & 200.6 & 22.4 \\
\hline $12 \mathrm{R}-6,16-19$ & 114.52 & 1.2010 & 1.0350 & 1.1610 & 196.9 & 40.3 & 48.8 & 140.6 & 9.0 & 238.1 & 39.7 \\
\hline $13 R-1,60-63$ & 117.80 & 1.2050 & 1.0450 & 1.1530 & 204.8 & 13.3 & 17.8 & 113.5 & 28.7 & 255.8 & 55.2 \\
\hline 13R-1, 94-97 & 118.14 & 1.2670 & 1.0810 & 1.1720 & 211.0 & 32.3 & 27.4 & 130.5 & 15.4 & 246.5 & 57.9 \\
\hline $13 \mathrm{R}-2,80-83$ & 119.37 & 1.2360 & 1.0520 & 1.1740 & 223.9 & 358.5 & 3.0 & 91.4 & 44.5 & 265.5 & 45.4 \\
\hline $13 \mathrm{R}-2,95-98$ & 119.52 & 1.1457 & 1.0335 & 1.1085 & 213.0 & 260.2 & 33.3 & 5.2 & 21.5 & 121.9 & 48.7 \\
\hline $13 \mathrm{R}-2,99-102$ & 119.56 & 1.2257 & 1.0238 & 1.1973 & 228.0 & 41.8 & 1.6 & 310.3 & 42.4 & 133.5 & 47.6 \\
\hline $13 R-3,36-39$ & 120.25 & 1.1020 & 1.0060 & 1.0960 & 230.4 & 71.5 & 21.2 & 333.2 & 20.4 & 203.3 & 59.9 \\
\hline $13 R-4,23-26$ & 121.51 & 1.0330 & 1.0160 & 1.0170 & 5.7 & 154.7 & 22.5 & 32.1 & 52.3 & 257.7 & 28.4 \\
\hline \multicolumn{12}{|l|}{ 153-920D- } \\
\hline $2 \mathrm{R}-1,21-24$ & 8.21 & 1.1270 & 1.0360 & 1.0890 & 134.3 & -4.2 & 20.6 & 123.1 & 58.2 & -103.4 & 23.1 \\
\hline $2 \mathrm{R}-1,24-27$ & 8.24 & 1.1130 & 1.0530 & 1.0570 & 118.2 & 192.7 & 12.0 & 89.0 & 48.1 & -67.3 & 39.4 \\
\hline $2 \mathrm{R}-1,116-119$ & 9.16 & 1.0820 & 1.0490 & 1.0320 & 164.2 & -84.1 & 73.5 & 14.4 & 2.5 & 105.1 & 16.3 \\
\hline $3 R-2,49-52$ & 19.49 & 1.1470 & 1.0310 & 1.1120 & 170.6 & 45.3 & 17.7 & -46.6 & 7.0 & -157.6 & 71.0 \\
\hline $4 \mathrm{R}-1,22-25$ & 27.52 & 1.1630 & 1.0990 & 1.0590 & 222.8 & 9.3 & 16.0 & -81.2 & 1.7 & -176.9 & 73.9 \\
\hline $4 \mathrm{R}-2,17-20$ & 28.77 & 1.1040 & 1.0500 & 1.0520 & 214.2 & 71.8 & 72.0 & -30.2 & 4.0 & -21.2 & 17.0 \\
\hline $4 \mathrm{R}-2,43-46$ & 29.03 & 1.1040 & 1.0434 & 1.0582 & 318.0 & -139.2 & 35.8 & -29.7 & 24.8 & 86.8 & 43.9 \\
\hline $4 \mathrm{R}-3,35-38$ & 30.19 & 1.1900 & 1.0080 & 1.1800 & 207.4 & 23.5 & 28.6 & 157.9 & 52.0 & -79.7 & 22.7 \\
\hline $5 \mathrm{R}-1.56-59$ & 37.46 & 1.1560 & 1.1010 & 1.0500 & & & & & & & \\
\hline $5 R-2,33-36$ & 38.24 & 1.0810 & 1.0120 & 1.0690 & 188.2 & & & & & & \\
\hline $5 \mathrm{R}-2,36-39$ & 38.27 & 1.1410 & 1.0190 & 1.1190 & 188.2 & 42.4 & 17.8 & -51.8 & 12.7 & -175.5 & 67.9 \\
\hline $5 \mathrm{R}-3,20-23$ & 39.47 & 1.0940 & 1.0890 & 1.0050 & 265.3 & -6.2 & 20.3 & 94.9 & 27.6 & -127.7 & 54.6 \\
\hline $5 R-3,127-130$ & 40.54 & 1.2700 & 1.0270 & 1.2360 & 205.9 & 6.5 & 13.5 & 101.2 & 18.8 & -117.1 & 66.5 \\
\hline $5 R-4,93-96$ & 41.66 & 1.1180 & 1.0660 & 1.0490 & 145.7 & 26.3 & 19.8 & -74.2 & 26.7 & 148.3 & 55.7 \\
\hline $6 \mathrm{R}-1,53-56$ & 47.03 & 1.1850 & 1.1390 & 1.0400 & 217.7 & 7.4 & 2.4 & 98.5 & 26.5 & -87.5 & 63.4 \\
\hline
\end{tabular}

\section{REFERENCES}

Bonatti, E., 1976. Serpentinite protrusions in the oceanic crust. Earth Planet. Sci. Lett., 32:107-113.

Cannat, M., 1993. Emplacement of mantle rocks in the seafloor at mid-ocean ridges. J. Geophys. Res., 98:4163-4172.

Cannat, M., Karson, J.A., Miller, D.J., et al., 1995. Proc, ODP, Init. Repts., 153: College Station, TX (Ocean Drilling Program).

Cannat, M., Mével, C., Maia, M., Deplus, C., Durand, C., Gente, P., Agrinier, P., Belarouchi, A., Dubuisson, G., et al., 1995. Thin crust, ultramafic exposures, and rugged faulting patterns at the Mid-Atlantic Ridge $\left(22^{\circ}-\right.$ $\left.24^{\circ} \mathrm{N}\right)$. Geology, 23:49-52.

Cannat, M., and Pariso, J., 1991. Partial reorientation of the deformational structures at Site 735 using paleodeclination measurements. In Von Herzen, R.P., Robinson, P.T., et al., Proc. ODP, Sci. Results, 118: College Station, TX (Ocean Drilling Program), 409-414.

Karson, J.A., 1990. Seafloor spreading on the Mid-Atlantic Ridge: implications for the structure of ophiolites and oceanic lithosphere produced in slow-spreading environments. In Malpas, J., Moores, E.M., Panayiotou, A., and Xenophontos, C. (Eds.), Ophiolites: Oceanic Crustal Analogues: Proc. Symp. "Troodos 1987": Nicosia, Cyprus (Minist. Agric. Nat. Resour.), 547-555.

Karson, J.A., and Dick, H.J.B., 1983. Tectonics of ridge-transform intersections at the Kane Fracture Zone. Mar. Geophys. Res., 6:51-98.

Kirschvink, J.L., 1980. The least-squares line and plane and the analysis of palaeomagnetic data. Geophys. J.R. Astron. Soc., 62:699-718.
McFadden, P.L., and Reid, A.B., 1982. Analysis of palaeomagnetic inclination data. Geophys. J.R. Astron. Soc., 69:307-319.

Schultz, N.J., Detrick, R.S., and Miller, S.P., 1988. Two- and three-dimensional inversion of magnetic anomalies in the MARK area (Mid-Atlantic Ridge, 23 N). Mar. Geophys. Res., 10:41-57.

Shipboard Scientific Party, 1995a. Explanatory notes. In Cannat, M., Karson, J.A., Miller, D.J., et al., Proc. ODP, Init. Repts., 153: College Station, TX (Ocean Drilling Program), 15-42.

, 1995b. Site 920. In Cannat, M., Karson, J.A., Miller, D.J., et al., Proc. ODP, Init. Repts., 153: College Station, TX (Ocean Drilling Program), 45-119.

, 1995c. Site 921. In Cannat, M., Karson, J.A., Miller, D.J., et al., Proc. ODP, Init. Repts., 153: College Station, TX (Ocean Drilling Program), 121-177.

, 1995d. Site 923. In Cannat, M., Karson, J.A., Miller, D.J., et al., Proc. ODP, Init. Repts., 153: College Station, TX (Ocean Drilling Program), 217-258.

Stacey, F.D., and Banerjee, S.K., 1974. The Physical Principles of Rock Magnetism. Dev. Solid Earth Geophys., 5: New York (Elsevier).

Date of initial receipt: 1 August 1995

Date of acceptance: 2 May 1996

Ms 153SR-040 
Table 3 (continued).

\begin{tabular}{|c|c|c|c|c|c|c|c|c|c|c|c|}
\hline \multirow[b]{2}{*}{$\begin{array}{l}\text { Core, section, } \\
\text { interval }(\mathrm{cm})\end{array}$} & \multirow[b]{2}{*}{$\begin{array}{l}\text { Depth } \\
\text { (mbsf) }\end{array}$} & \multicolumn{3}{|c|}{ AMS } & \multirow[b]{2}{*}{$\begin{array}{l}\text { Char. declin. } \\
\text { (uncorr.) }\end{array}$} & \multicolumn{6}{|c|}{ AMS directions, reoriented } \\
\hline & & $K_{1} / K_{3}$ & $K_{1} / K_{2}$ & $K_{2} / K_{3}$ & & $\begin{array}{c}K_{l} \\
\text { declin. }\end{array}$ & $\begin{array}{c}K_{l} \\
\text { inclin. }\end{array}$ & $\begin{array}{c}K_{2} \\
\text { declin. }\end{array}$ & $\begin{array}{c}K_{2} \\
\text { inclin. }\end{array}$ & $\begin{array}{c}K_{3} \\
\text { declin. }\end{array}$ & $\begin{array}{c}K_{3} \\
\text { inclin. }\end{array}$ \\
\hline \multicolumn{12}{|l|}{ 153-920D- } \\
\hline $6 \mathrm{R}-2,48-51$ & 48.30 & 1.1480 & 1.0100 & 1.1360 & 273.3 & -32.7 & 20.1 & 100.3 & 61.8 & -129.9 & 19.0 \\
\hline $6 \mathrm{R}-3,54-57$ & 49.60 & 1.1410 & 1.0700 & 1.0660 & 200.2 & 3.5 & 40.7 & -118.3 & 31.5 & 127.9 & 33.3 \\
\hline $8 \mathrm{R}-1,141-144$ & 67.31 & 1.2720 & 1.0220 & 1.2450 & 4.0 & 159.4 & 14.2 & 12.2 & 73.3 & 251.7 & 8.7 \\
\hline $8 R-2,43-46$ & 67.77 & 1.1360 & 1.0360 & 1.0970 & 167.2 & -8.0 & 6.0 & 116.6 & 79.5 & -98.9 & 8.5 \\
\hline $10 \mathrm{R}-2,87-90$ & 80.36 & 1.1333 & 1.0841 & 1.0454 & 186.0 & -150.0 & 82.1 & 30.6 & 7.9 & -59.4 & 0.1 \\
\hline $10 \mathrm{R}-2,90-93$ & 80.39 & 1.1710 & 1.0590 & 1.1058 & 194.0 & -162.8 & 71.2 & 13.7 & 18.8 & 104.0 & 1.1 \\
\hline 10R-2, 134-137 & 80.83 & 1.1010 & 1.0250 & 1.0740 & 202.1 & -5.1 & 2.9 & 89.0 & 55.0 & -97.1 & 34.8 \\
\hline IOR-3, 74-77 & 81.70 & 1.1950 & 1.0300 & 1.1600 & 201.5 & 6.92 & 9.61 & 60.9 & 57.7 & -89.9 & 11.8 \\
\hline $10 \mathrm{R}-3,77-80$ & 81.73 & 1.2020 & 1.0190 & 1.1800 & 201.5 & & & & & & \\
\hline $10 \mathrm{R}-4,80-83$ & 83.24 & 1.2220 & 1.0800 & 1.1320 & 233.2 & 4.1 & 38.4 & 112.9 & 22.1 & -134.6 & 43.4 \\
\hline $11 R-1,27-30$ & 85.57 & 1.2180 & 1.0350 & 1.1770 & 37.6 & 0.3 & 5.8 & 93.5 & 29.2 & 260.2 & 60.1 \\
\hline $11 \mathrm{R}-1,125-128$ & 86.55 & 1.2640 & 1.0520 & 1.2020 & 198.6 & 173.6 & 7.46 & 7.46 & 5.0 & -93.2 & 23.7 \\
\hline $11 \mathrm{R}-2,43-46$ & 87.15 & 1.1685 & 1.0747 & 1.0873 & 223.0 & -178.5 & 38.4 & 0.1 & 51.6 & 90.9 & 0.7 \\
\hline $11 R-2,46-49$ & 87.18 & 1.2450 & 1.0745 & 1.1587 & 225.0 & 179.04 & 1.5 & -16.2 & 47.5 & 82.2 & 7.6 \\
\hline $11 R-2,63-66$ & 87.35 & 1.2010 & 1.0330 & 1.1620 & 223.7 & 21.8 & 30.6 & 127.1 & 24.0 & -111.7 & 49.3 \\
\hline $11 R-2,66-69$ & 87.38 & 1.2160 & 1.0190 & 1.1930 & 223.7 & & & & & & \\
\hline $11 R-3,6-9$ & 87.98 & 1.2395 & 1.0111 & 1.2259 & 215.0 & -162.6 & 33.1 & -25.8 & 48.2 & 91.7 & 22.4 \\
\hline $11 \mathrm{R}-3,9-12$ & 88.01 & 1.1960 & 1.0103 & 1.1838 & 206.0 & 41.7 & 45.0 & 178.6 & 36.1 & -73.4 & 23.0 \\
\hline $11 R-3,22-25$ & 88.14 & 1.2340 & 1.0500 & 1.1750 & 222.3 & & & & & & \\
\hline $11 \mathrm{R}-3,25-28$ & 88.17 & 1.1730 & 1.0260 & 1.1430 & 222.3 & 72.0 & 53.9 & 164.8 & 2.0 & -103.8 & 36.0 \\
\hline $11 \mathrm{R}-3,49-52$ & 88.41 & 1.1123 & 1.0050 & 1.1067 & 192.0 & -109.4 & 71.0 & 10.0 & 9.6 & 102.8 & 16.3 \\
\hline $11 \mathrm{R}-3,52-55$ & 88.44 & 1.2071 & 1.0139 & 1.1905 & 192.0 & -153.4 & 14.2 & 25.3 & 75.8 & 116.6 & 0.3 \\
\hline $12 \mathrm{R}-1,104-107$ & 96.04 & 1.2590 & 1.0770 & 1.1690 & 181.7 & 159.9 & 3.9 & 67.7 & 29.1 & -103.3 & 60.6 \\
\hline $12 \mathrm{R}-2,119-122$ & 97.64 & 1.2620 & 1.0380 & 1.2160 & 205.2 & 151.9 & 25.0 & 38.1 & 40.9 & -96.1 & 38.8 \\
\hline $12 R-3,65-68$ & 98.38 & 1.2899 & 1.0494 & 1.2292 & 241.0 & -163.2 & 30.6 & -36.6 & 45.3 & 87.6 & 29.1 \\
\hline $12 \mathrm{R}-3,68-70$ & 98.41 & 1.2932 & 1.0373 & 1.2466 & 229.0 & -156.1 & 48.3 & -4.5 & 38.1 & 97.1 & 14.4 \\
\hline $12 \mathrm{R}-3,86-89$ & 98.59 & 1.2400 & 1.0730 & 1.1550 & 246.2 & -2.9 & 14.8 & 98.6 & 37.2 & -110.6 & 49.0 \\
\hline $12 \mathrm{R}-4,54-57$ & 99.57 & 1.1190 & 1.0350 & 1.0820 & 219.1 & -22.2 & 3.9 & 71.3 & 41.1 & -116.6 & 48.6 \\
\hline $12 R-4,103-106$ & 100.06 & 1.2818 & 1.1052 & 1.1598 & 234.0 & -107.3 & 33.4 & 161.3 & 2.2 & 68.0 & 56.5 \\
\hline $12 R-4,107-110$ & 100.10 & 1.2504 & 1.0995 & 1.1372 & 236.0 & -112.4 & 32.1 & 155.8 & 2.9 & 61.2 & 57.7 \\
\hline $12 \mathrm{R}-5,26-29$ & 100.79 & 1.3200 & 1.0660 & 1.2370 & 120.2 & 162.7 & 20.9 & 48.9 & 46.7 & -91.2 & 35.9 \\
\hline $13 \mathrm{R}-2,84-87$ & 106.33 & 1.2130 & 1.1590 & 1.0470 & 270.1 & -2.6 & 9.1 & 104.4 & 61.4 & -97.2 & 26.9 \\
\hline $13 \mathrm{R}-3,126-129$ & 108.19 & 1.2660 & 1.0100 & 1.2540 & 175.6 & 28.7 & 21.0 & 121.5 & 7.3 & -130.4 & 67.7 \\
\hline $13 R-4,41-44$ & 108.74 & 1.1430 & 1.0730 & 1.0650 & 188.5 & -170.4 & 10.6 & 92.0 & 35.0 & -66.1 & 53.0 \\
\hline $14 \mathrm{R}-1,58-61$ & 114.88 & 1.2170 & 1.1220 & 1.0860 & 196.3 & 7.9 & 9.2 & -86.8 & 26.9 & 115.1 & 61.4 \\
\hline $14 \mathrm{R}-2,35-38$ & 116.14 & 1.2359 & 1.0553 & 1.1711 & 220.0 & & & & & & \\
\hline $14 \mathrm{R}-2,38-41$ & 116.17 & 1.2766 & 1.0398 & 1.2277 & 220.0 & -177.3 & 45.4 & 2.9 & 44.6 & -87.2 & 0.1 \\
\hline $14 \mathrm{R}-3,82-85$ & 117.85 & 1.1430 & 1.0450 & 1.0940 & 109.5 & 14.7 & 23.1 & 108.9 & 9.8 & 220.2 & 64.7 \\
\hline $14 \mathrm{R}-3,91-94$ & 117.94 & 1.1439 & 1.0727 & 1.0664 & 338.0 & & & & & & \\
\hline $14 \mathrm{R}-3,94-97$ & 117.97 & 1.1737 & 1.0530 & 1.1147 & 338.0 & -78.0 & 63.4 & 149.4 & 18.7 & 53.0 & 18.2 \\
\hline $14 \mathrm{R}-4,137-140$ & 119.86 & 1.2800 & 1.1970 & 1.0690 & 159.8 & -16.5 & 14.1 & 157.3 & 75.9 & -106.9 & 1.5 \\
\hline $14 \mathrm{R}-5,69-72$ & 120.59 & 1.3180 & 1.0040 & 1.3120 & 212.0 & 163.3 & 15.4 & 10.2 & 72.8 & -104.7 & 7.4 \\
\hline $14 \mathrm{R}-5,106-109$ & 120.96 & 1.1599 & 1.0149 & 1.1429 & 50.0 & 71.4 & 57.4 & 281.6 & 28.9 & 183.8 & 13.7 \\
\hline $14 R-5,109-112$ & 120.99 & 1.2623 & 1.0289 & 1.2268 & 55.0 & 72.5 & 44.3 & 294.9 & 37.1 & 186.5 & 22.6 \\
\hline $15 \mathrm{R}-1,80-83$ & 124.70 & 1.3030 & 1.0310 & 1.2640 & 195.0 & 156.7 & 13.3 & 51.1 & 48.8 & -102.6 & 38.1 \\
\hline $15 R-2,92-95$ & 126.04 & 1.2030 & 1.0330 & 1.1640 & 213.7 & -1.4 & 10.8 & 101.1 & 48.5 & -100.4 & 39.5 \\
\hline $15 R-3,48-51$ & 126.99 & 1.2120 & 1.0660 & 1.1380 & 237.6 & 14.7 & 27.4 & 144.6 & 51.0 & -89.5 & 25.4 \\
\hline $15 R-3,93-96$ & 127.44 & 1.2732 & 1.0307 & 1.2353 & 206.0 & -156.3 & 55.6 & 1.0 & 32.3 & 97.8 & 10.6 \\
\hline
\end{tabular}
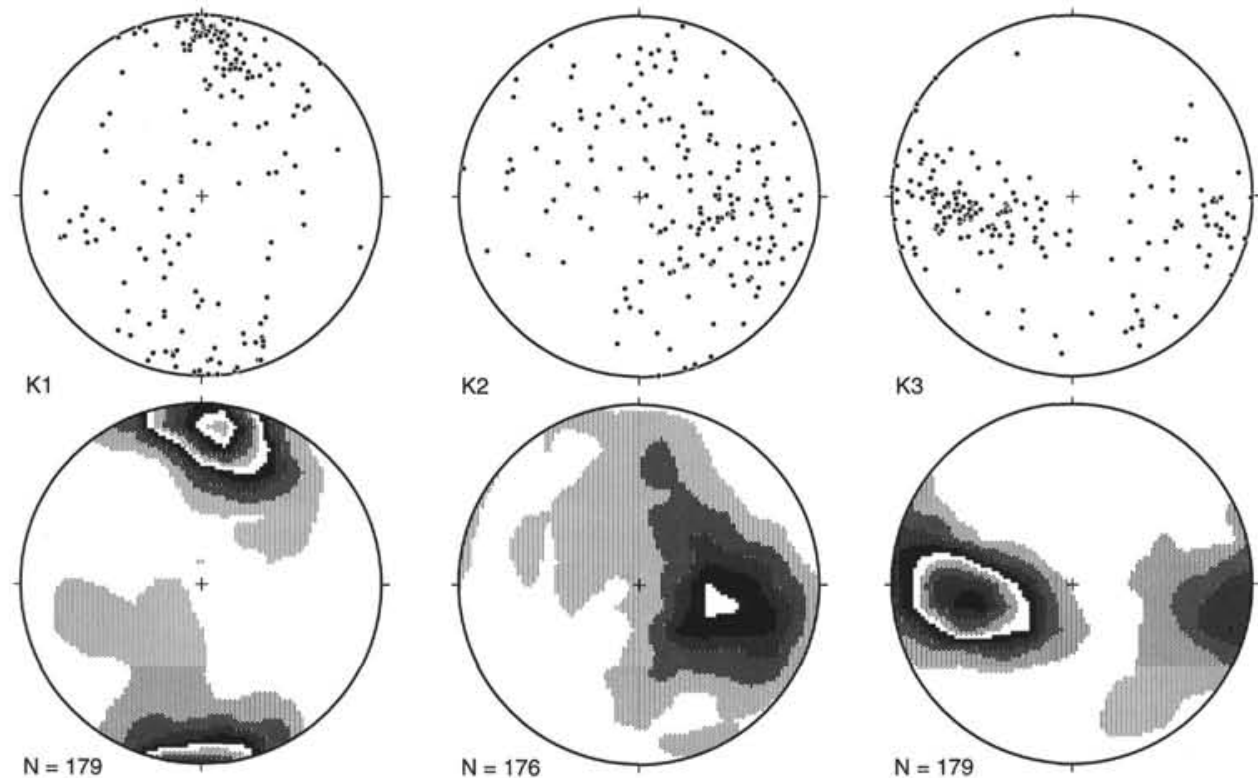

Figure 4. Lower hemisphere stereographic projection plots of the principal axes of the susceptibility ellipsoid after reorientation. The minimum susceptibility axis $\left(K_{3}\right)$ dips about $30^{\circ}$ to the west-southwest, and therefore the magnetic foliation dips to the east-northeast, approximately parallel to the orientation of the V2 vein set and the crystal-plastic fabric. 
Table 3 (continued).

\begin{tabular}{|c|c|c|c|c|c|c|c|c|c|c|c|}
\hline \multirow[b]{2}{*}{$\begin{array}{l}\text { Core, section, } \\
\text { interval }(\mathrm{cm})\end{array}$} & \multirow[b]{2}{*}{$\begin{array}{l}\text { Depth } \\
\text { (mbsf) }\end{array}$} & \multicolumn{3}{|c|}{ AMS } & \multirow[b]{2}{*}{$\begin{array}{l}\text { Char. declin. } \\
\text { (uncorr.) }\end{array}$} & \multicolumn{6}{|c|}{ AMS directions, reoriented } \\
\hline & & $K_{1} / K_{3}$ & $K_{1} / K_{2}$ & $K_{2} / K_{3}$ & & $\begin{array}{c}K_{l} \\
\text { declin. }\end{array}$ & $\begin{array}{c}K_{l} \\
\text { inclin. }\end{array}$ & $\begin{array}{c}K_{2} \\
\text { declin. }\end{array}$ & $\begin{array}{c}K_{2} \\
\text { inclin. }\end{array}$ & $\begin{array}{c}K_{3} \\
\text { declin. }\end{array}$ & $\begin{array}{c}K_{3} \\
\text { inclin. }\end{array}$ \\
\hline $15 \mathrm{R}-3,96-99$ & 127.47 & 1.1984 & 1.0266 & 1.1674 & 206.0 & & & & & & \\
\hline $15 R-4,72-75$ & 128.47 & 1.3160 & 1.0430 & 1.2620 & 208.7 & -2.0 & 12.8 & 104.1 & 50.9 & -101.6 & 36.2 \\
\hline $15 R-6,76-79$ & 131.11 & 1.1579 & 1.0505 & 1.1022 & 295.0 & & & & & & \\
\hline $15 \mathrm{R}-6,79-82$ & 131.14 & 1.1968 & 1.0699 & 1.1186 & 295.0 & -65.1 & 41.6 & -172.0 & 18.1 & 80.3 & 42.8 \\
\hline $16 \mathrm{R}-1,98-101$ & 134.38 & 1.1730 & 1.0880 & 1.0780 & 206.2 & & & & & & \\
\hline $16 \mathrm{R}-1,101-104$ & 134.41 & 1.1380 & 1.1080 & 1.0270 & 206.2 & 12.7 & 26.5 & 106.3 & 7.2 & -149.8 & 62.4 \\
\hline $16 \mathrm{R}-3,5-8$ & 135.97 & 1.0810 & 1.0400 & 1.0390 & 249.6 & 9.7 & 4.3 & 105.2 & 52.5 & -83.6 & 37.2 \\
\hline $16 R-4,20-23$ & 137.18 & 1.0911 & 1.0140 & 1.0761 & 186.0 & -104.1 & 43.0 & 33.2 & 38.2 & 142.6 & 22.9 \\
\hline $16 \mathrm{R}-4,25-28$ & 137.23 & 1.1225 & 1.0076 & 1.1141 & 184.0 & 8.5 & 66.3 & -109.9 & 11.8 & 155.7 & 20.3 \\
\hline $16 \mathrm{R}-4,82-85$ & 137.80 & 1.3380 & 1.0310 & 1.2970 & 189.2 & 22.0 & 6.8 & 113.7 & 14.0 & -93.2 & 74.4 \\
\hline $16 \mathrm{R}-5,80-83$ & 138.93 & 1.2350 & 1.0500 & 1.1760 & 223.8 & -18.7 & 4.8 & 74.2 & 32.1 & -116.2 & 57.5 \\
\hline $16 \mathrm{R}-7,54-57$ & 141.53 & 1.2370 & 1.0640 & 1.1630 & 278.0 & & & & & & \\
\hline $16 \mathrm{R}-7,57-60$ & 141.56 & 1.2370 & 1.0730 & 1.1530 & 278.0 & 160.7 & 4.2 & 68.0 & 32.2 & -102.7 & 57.5 \\
\hline $17 \mathrm{R}-3,37-40$ & 145.74 & 1.1610 & 1.0430 & 1.1130 & 222.0 & & & & & (5). & \\
\hline 17R- $3,40-43$ & 145.77 & 1.1630 & 1.0430 & 1.1150 & 222.0 & 107.8 & 8.2 & 4.9 & 57.3 & -157.2 & 31.4 \\
\hline $17 R-4,103-106$ & 147.69 & 1.3110 & 1.0480 & 1.2520 & 228.8 & 6.3 & 21.6 & 109.7 & 30.2 & -113.5 & 51.4 \\
\hline $18 \mathrm{R}-1,123-126$ & 153.83 & 1.1040 & 1.0150 & 1.0880 & 344.0 & 146.8 & 33.5 & -12.4 & 54.7 & -116.6 & 9.8 \\
\hline $18 \mathrm{R}-2,70-73$ & 154.80 & 1.1526 & 1.0375 & 1.1109 & 296.0 & -54.4 & 33.3 & -175.7 & 38.3 & 62.0 & 34.1 \\
\hline $18 \mathrm{R}-2,83-87$ & 154.93 & 1.1683 & 1.0419 & 1.1214 & 293.0 & -48.4 & 32.6 & -173.4 & 41.9 & 64.1 & 30.9 \\
\hline $18 \mathrm{R}-3,75-78$ & 156.10 & 1.1590 & 1.0400 & 1.1140 & 198.2 & -40.7 & 2.0 & 17.07 & 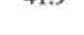 & 6.1. & (30. \\
\hline $18 \mathrm{R}-3,78-81$ & 156.13 & 1.1260 & 1.0660 & 1.0570 & 198.2 & 2.6 & 9.9 & 107.9 & 56.5 & -93.6 & 31.6 \\
\hline $18 \mathrm{R}-4,12-15$ & 156.85 & 1.2040 & 1.1100 & 1.0840 & 190.8 & 13.4 & 8.6 & 107.2 & 23.9 & -95.0 & 64.4 \\
\hline $19 \mathrm{R}-1,18-21$ & 162.48 & 1.1580 & 1.1170 & 1.1580 & 212.8 & -4.5 & 20.4 & 92.1 & 17.3 & -140.8 & 62.7 \\
\hline $19 R-2,13-16$ & 163.80 & 1.1980 & 1.0760 & 1.1130 & 195.2 & - & 20.7 & 2.1. & . & 170.0 & \\
\hline $19 \mathrm{R}-2,16-19$ & 163.83 & 1.1880 & 1.0660 & 1.1150 & 195.2 & -0.2 & 11.1 & 101.8 & 46.6 & -100.1 & 41.3 \\
\hline $20 \mathrm{R}-1,103-106$ & 172.93 & 1.2130 & 1.1260 & 1.0780 & 197.2 & 17.7 & 23.6 & 117.2 & 20.7 & -116.1 & 57.8 \\
\hline $20 \mathrm{R}-1,106-109$ & 172.96 & 1.2040 & 1.1280 & 1.0680 & 197.2 & & & & & & \\
\hline $20 \mathrm{R}-2,24-27$ & 173.30 & 1.1960 & 1.1240 & 1.0640 & 200.9 & 10.0 & 24.3 & 110.0 & 21.1 & -123.7 & 56.9 \\
\hline $20 \mathrm{R}-3,80-83$ & 174.93 & 1.2320 & 1.0340 & 1.1910 & 247.8 & -4.1 & 17.8 & 118.3 & 59.0 & -102.5 & 24.4 \\
\hline $20 \mathrm{R}-4,38-41$ & 175.92 & 1.1800 & 1.0430 & 1.1310 & 202.7 & -0.5 & 12.5 & 102.5 & 45.5 & -102.0 & 41.8 \\
\hline $20 R-4,41-44$ & 175.95 & 1.1320 & 1.0980 & 1.0320 & 202.7 & & & & & & \\
\hline $20 \mathrm{R}-5,75-78$ & 177.75 & 1.2387 & 1.0893 & 1.1371 & 174.0 & -54.7 & 78.9 & 65.1 & 5.6 & 156.0 & 9.5 \\
\hline $20 \mathrm{R}-5,78-82$ & 177.78 & 1.2417 & 1.0711 & 1.1592 & 185.0 & -46.7 & 77.7 & 49.3 & 1.3 & 139.6 & 12.2 \\
\hline $20 \mathrm{R}-5,98-101$ & 177.98 & 1.2480 & 1.0800 & 1.1560 & 188.8 & 177.5 & 9.0 & 82.4 & 29.2 & -77.1 & 59.2 \\
\hline $21 R-1,93-96$ & 182.43 & 1.3740 & 1.0610 & 1.2950 & $\begin{array}{l}205.9 \\
205.9\end{array}$ & 0.2 & 3.9 & 94.5 & 47.4 & -93.4 & 42.3 \\
\hline $21 \mathrm{R}-2,40-43$ & 183.15 & 1.2950 & 1.0580 & 1.2240 & 196.7 & -2.6 & 5.5 & 90.6 & 30.1 & -102.0 & 59.3 \\
\hline $21 R-2,43-46$ & 183.18 & 1.2530 & 1.0510 & 1.1920 & 196.7 & & & & & & \\
\hline $21 R-2,97-100$ & 183.72 & 1.2720 & 1.2310 & 1.0330 & 170.6 & -7.3 & 4.1 & 85.1 & 31.0 & -104.0 & 58.7 \\
\hline $21 \mathrm{R}-3,22-25$ & 184.32 & 1.3110 & 1.0350 & 1.2660 & 232.2 & 159.9 & 3.6 & 68.5 & 21.5 & -101.1 & 68.2 \\
\hline $21 R-4,68-71$ & 186.28 & 1.2040 & 1.0320 & 1.1670 & 222.2 & 15.5 & 11.2 & 113.3 & 34.2 & -90.1 & 53.5 \\
\hline $22 \mathrm{R}-2,27-30$ & 192.07 & 1.1217 & 1.0697 & $\begin{array}{l}1.0487 \\
\end{array}$ & 249.0 & -171.5 & 13.7 & -53.2 & 62.8 & 92.6 & 23.1 \\
\hline $22 \mathrm{R}-2,30-33$ & 192.10 & 1.1032 & 1.0665 & 1.0344 & 250.0 & -171.7 & 4.3 & -79.5 & 26.9 & 89.9 & 62.7 \\
\hline $22 \mathrm{R}-2,86-89$ & 192.66 & 1.1900 & 1.0480 & 1.1350 & 260.9 & & & & & & \\
\hline $22 \mathrm{R}-2,89-92$ & 192.69 & 1.2110 & 1.0530 & 1.1510 & 260.9 & 4.5 & 38.9 & 178.7 & 50.9 & -87.8 & 2.9 \\
\hline $22 R-3,17-20$ & 192.90 & 1.0580 & 1.0300 & 1.0280 & 215.6 & 6.8 & 9.7 & 126.4 & 70.9 & -86.0 & 16.3 \\
\hline $22 \mathrm{R}-4,50-53$ & 193.93 & 1.2350 & 1.0740 & 1.1500 & 170.4 & 176.3 & 9.4 & 83.1 & 18.2 & -67.5 & 69.4 \\
\hline $22 \mathrm{R}-5,104-107$ & 195.67 & 1.3420 & 1.0740 & 1.2500 & 201.1 & 3.2 & 7.1 & 94.9 & 13.6 & -113.8 & 74.5 \\
\hline $22 \mathrm{R}-5,107-110$ & 195.70 & 1.3160 & 1.0820 & 1.2170 & 204.6 & 2.0 & 12.1 & 94.8 & 12.8 & -129.9 & 72.2 \\
\hline $22 \mathrm{R}-6,24-26$ & 196.28 & 1.3485 & 1.0656 & 1.2655 & 218.0 & -139.5 & 51.5 & 41.1 & 38.5 & -49.1 & 0.3 \\
\hline $22 \mathrm{R}-6,26-29$ & 196.30 & 1.3086 & 1.0617 & 1.2326 & 228.0 & -155.1 & 65.6 & 29.2 & 24.3 & -61.5 & 1.6 \\
\hline $22 \mathrm{R}-6,15-18$ & 196.35 & 1.2840 & 1.0510 & 1.2220 & 234.0 & & & & & & \\
\hline
\end{tabular}

Notes: The correction factor is equal to $360^{\circ}-\mathrm{D}$ (the uncorrected stable remanent declination). Therefore, the corrected orientations are in the system where north is parallel to the remanent declination. Char. declin.$=$ characteristic declination, inclin.$=$ inclination, uncorr,$=$ uncorrected, corr,$=$ corrected

Figure 5. Diagram showing the change in both inclination and declination for a unit vector with orientation of $0^{\circ}$ inclination and $41^{\circ}$ declination when rotated about horizontal axes with azimuths $\pm 20^{\circ}$ about north. The solid circle shows the original orientation of the vector and the open circle denotes its orientation after a $14^{\circ}$ clockwise rotation about a horizontal axis with a $20^{\circ}$ trend or a similar counterclockwise rotation about an axis with a $340^{\circ}$ trend. The gray area is the $95 \%$ confidence interval for the inclination-only mean for the measured samples. Modified from figure 94, Shipboard Scientific Party (1995b).

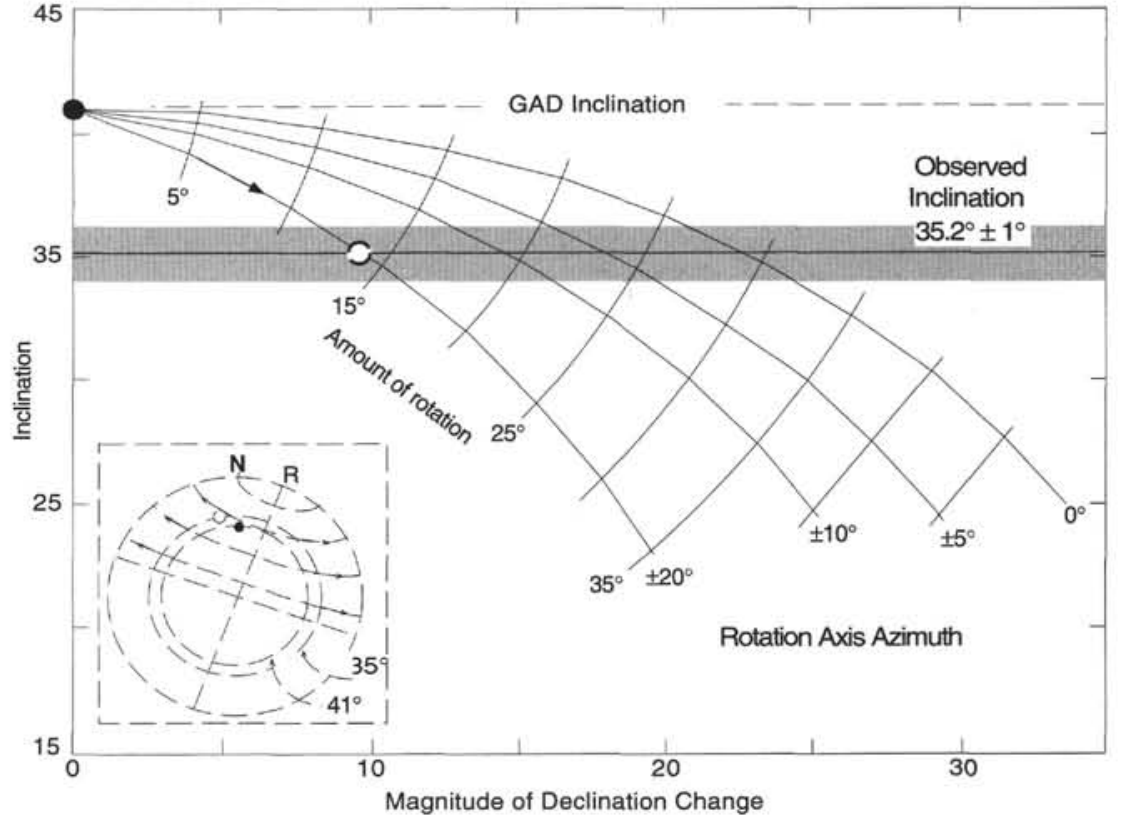


A

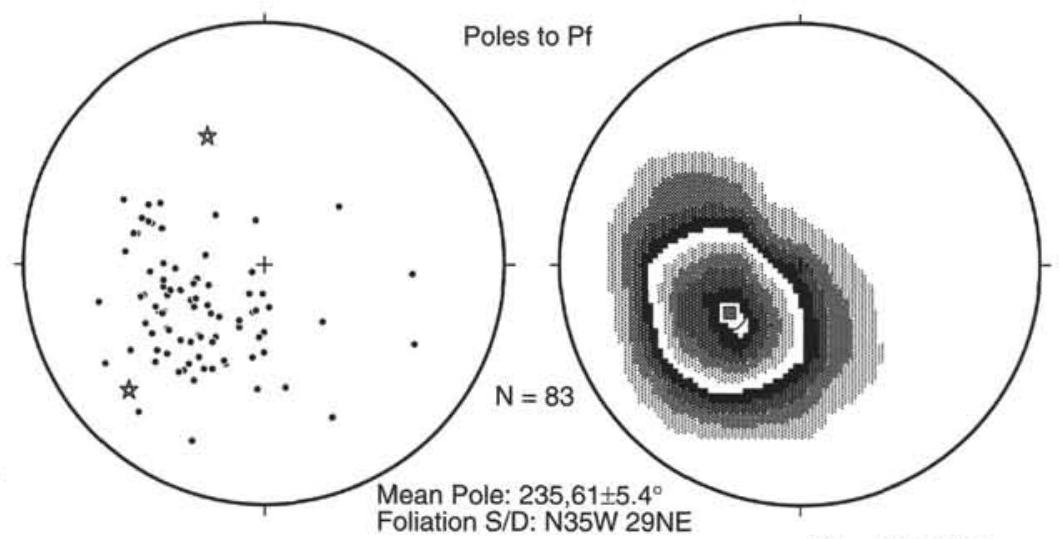

Foliation S/D: N35W 29NE

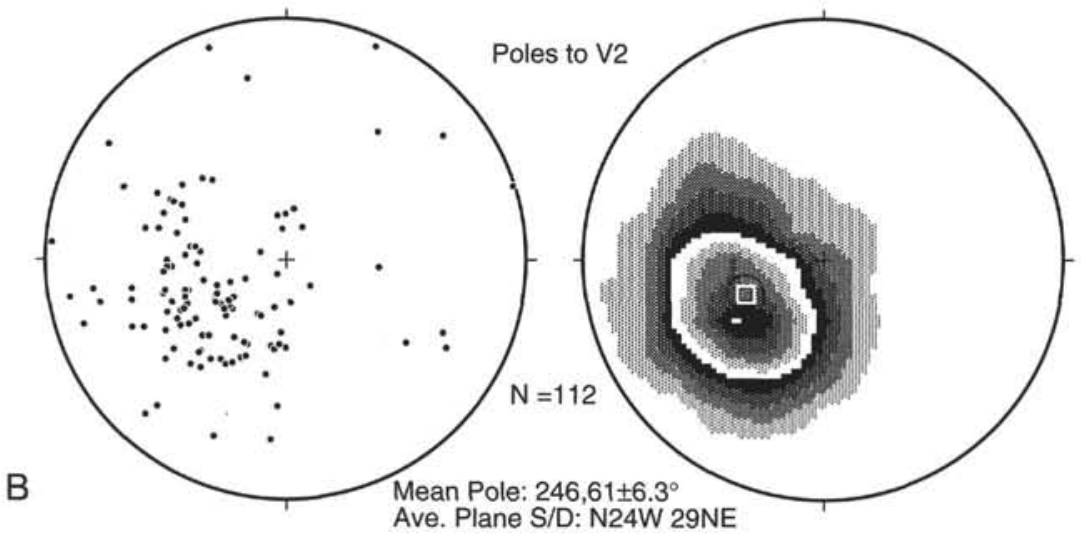

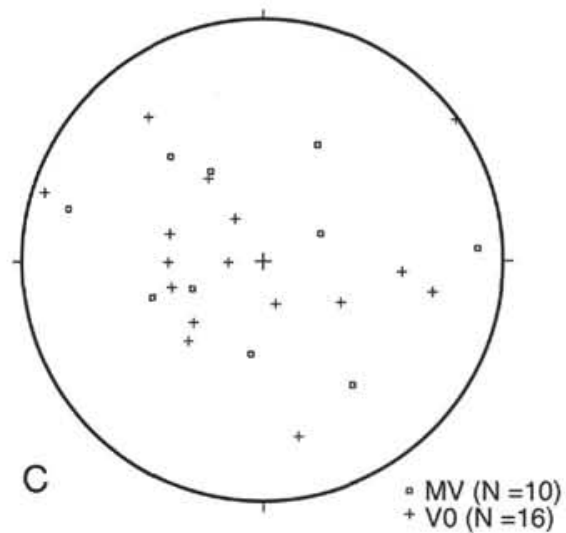

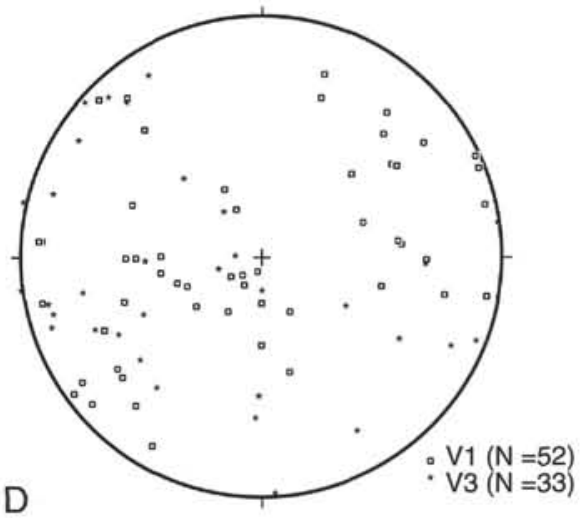

Figure 6. Stereographic projections of the reconstructed orientation of the deformation structures (foliation, veins) in core from Holes 920B and 920D. The measured stable remanent magnetic declination was rotated to due north and orientations of features measured in the core reference frame appropriately restored. Combined measurements for both holes are shown on lower hemisphere equal-area stereographic projections. A. Scatter plot of poles to the plastic foliation defined by elongate remnants of orthopyroxene crystals, and accompanying Kamb contour plot. Also shown as stars are the stable remanent magnetic directions for two oriented samples collected by Alvin near Site 920 . B. Equivalent diagrams for the V2 generation of veins. C. Combined scatter plot of poles to magmatic veins (MV) and vein set V0. D. Combined scatter plot of poles to veins generations V1 and V3. Note that poles to the V3 vein set do not occur in the upper right quadrant, therefore the average V3 vein has a dip to the northeast, similar to the plastic foliation and the V2 vein set.

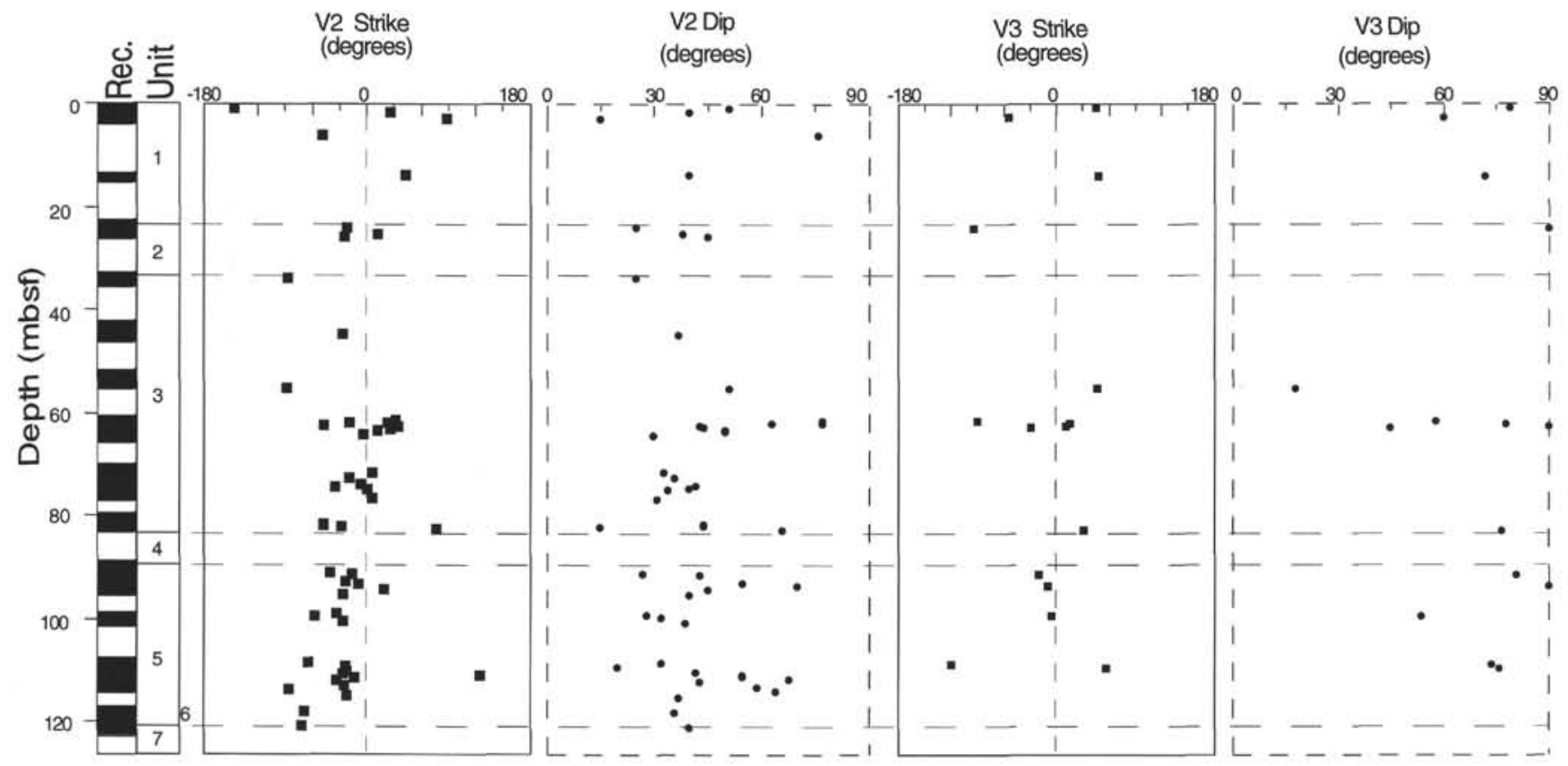

Figure 7. Downhole orientation variations for the V2 and V3 veins sets in Hole 920 . The average V2 strike is $343^{\circ}$ and the dip is $35^{\circ}$. Note that the orientation of the strike is generally more west trending at the deepest samples. Although the V3 vein set has many fewer corrected orientations measured, the average strike is near $0^{\circ}$, although $\mathrm{V} 3$ dips are generally much greater than those for $\mathrm{V} 2$ veins. Rec. $=$ recovery. 


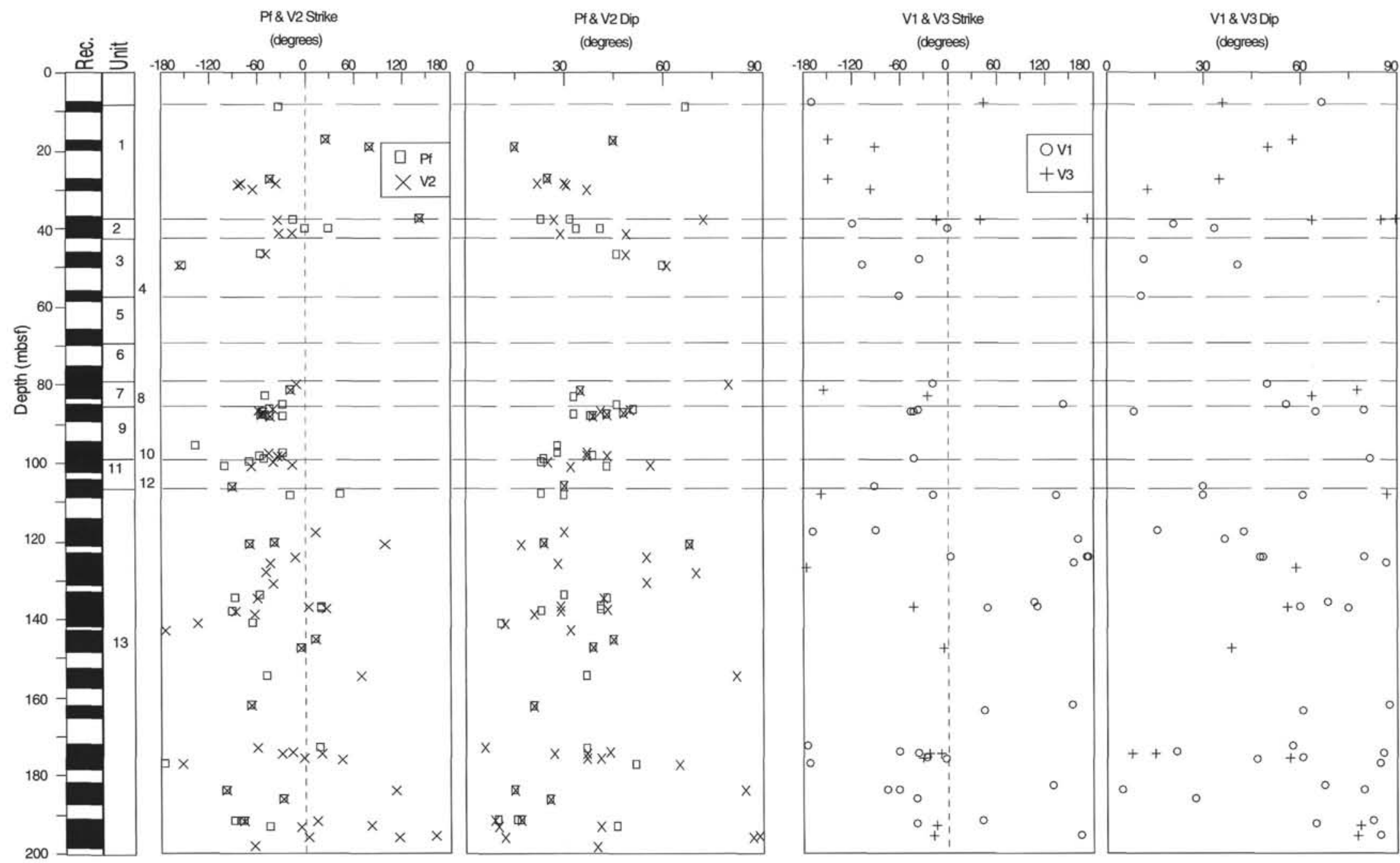

Figure 8. Downhole orientation variations for the plastic fabric (PF) and several vein sets in Hole 920D. The first two columns show strike and dip for PF and $\mathrm{V} 2$ combined. In general, these features are parallel, although near the bottom of the hole significant differences in both strike and dip are common. The mean orientation of $\mathrm{V} 2$ is $\mathrm{N} 33^{\circ} \mathrm{W}, 25^{\circ} \mathrm{NE}$, and of $\mathrm{PF}$ is $\mathrm{N} 41^{\circ} \mathrm{W}, 24^{\circ} \mathrm{NE}$. The V1 and V3 vein generations show no coherent variation in orientation with depth. Rec. $=$ recovery. 


\section{Poles to all reoriented structures}

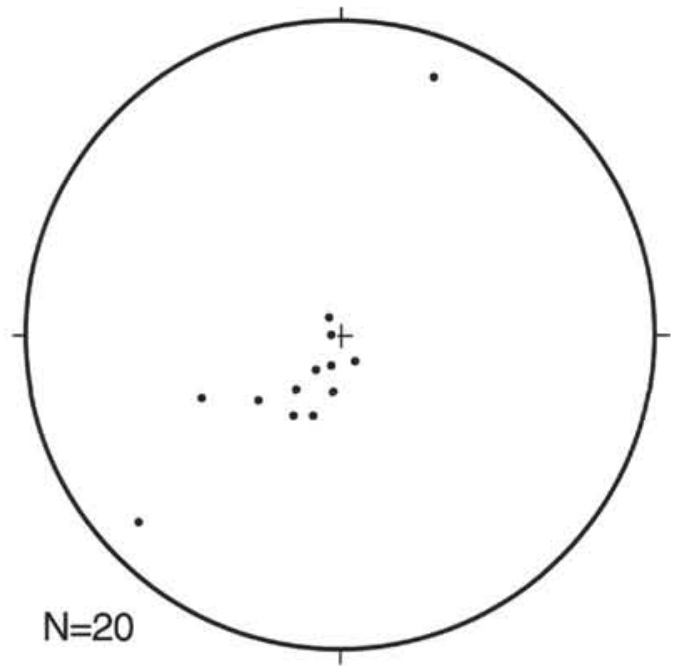

Figure 9. Equal-area stereographic projection of reoriented structures in core from Hole $923 \mathrm{~A}$. This hole was drilled to approximately $80 \mathrm{~m}$ with very high recovery.

Table 4. Reorientation data for samples from Hole 923A

\begin{tabular}{|c|c|c|c|c|c|}
\hline $\begin{array}{l}\text { Core, } \\
\text { section }\end{array}$ & Piece & $\begin{array}{c}\text { Char. declin. } \\
\left(^{\circ}\right) \\
\text { (uncorr.) }\end{array}$ & Feature & $\begin{array}{c}\text { Strike, dip } \\
\left({ }^{\circ}\right) \\
\text { (uncorr.) }\end{array}$ & $\begin{array}{c}\text { Strike, dip } \\
\left({ }^{\circ}\right) \\
\text { (corr.) }\end{array}$ \\
\hline $2 \mathrm{R}-1$ & 4 & 356 & PF & 147,47 & 151,47 \\
\hline $2 \mathrm{R}-1$ & 4 & 356 & V & 291,37 & 295,37 \\
\hline $2 \mathrm{R}-2$ & 1 & 18 & V5a & 347,39 & 329,39 \\
\hline $2 \mathrm{R}-2$ & 1 & 18 & SZ & 166,56 & 148,56 \\
\hline $2 \mathrm{R}-2$ & 1 & 18 & $\mathrm{~V} 4 \mathrm{a}$ & 30,49 & 12,49 \\
\hline $2 \mathrm{R}-2$ & 1 & 18 & J & 57.64 & 39,64 \\
\hline $2 \mathrm{R}-2$ & 1 & 18 & V4 & 45,76 & 27,76 \\
\hline $2 R-2$ & 1 & 18 & V5b & 10,40 & 352,40 \\
\hline $2 \mathrm{R}-2$ & 8 & 162 & PF & 305,46 & 143,46 \\
\hline $2 \mathrm{R}-2$ & 8 & 162 & MV & 225,59 & 63,59 \\
\hline $2 \mathrm{R}-2$ & 8 & 162 & V & 159,16 & 357,16 \\
\hline $3 R-1$ & $3 \mathrm{~A}$ & 90 & $\mathrm{Va}$ & 5,55 & 275,55 \\
\hline $3 R-1$ & $3 \mathrm{~A}$ & 90 & CTV & 168,41 & 78,41 \\
\hline $3 R-1$ & $3 \mathrm{~A}$ & 90 & PF & 120,49 & 30,49 \\
\hline $3 R-1$ & 3B & 90 & V & 76,21 & 346,21 \\
\hline $3 R-1$ & $5 \mathrm{~A}$ & 29 & $\mathrm{Vc}$ & 247.8 & 218,8 \\
\hline $3 R-1$ & $5 \mathrm{~A}$ & 29 & Pf & 131,30 & 102,30 \\
\hline $3 R-1$ & $5 \mathrm{~A}$ & 29 & $\mathrm{Va}$ & 90,44 & 61,44 \\
\hline $3 R-1$ & $5 B$ & 29 & CTV & 130,27 & 101,27 \\
\hline $3 \mathrm{R}-1$ & $5 \mathrm{C}$ & 29 & BS & 180,15 & 151,15 \\
\hline $3 R-1$ & 6 & 264 & CTV & 192,22 & 288,22 \\
\hline $3 R-1$ & 6 & 264 & CTV & 204,24 & 300,24 \\
\hline $3 R-1$ & 6 & 264 & PF & 213,18 & 309,18 \\
\hline $3 R-1$ & 6 & 264 & CTV & 225,27 & 321,27 \\
\hline $3 R-2$ & 2 & 54 & SZ & 206,45 & 152,45 \\
\hline $3 R-2$ & 2 & 54 & PF & 206,45 & 152,45 \\
\hline $4 \mathrm{R}-1$ & 7 & 84 & PF & 160,32 & 76,32 \\
\hline $4 \mathrm{R}-1$ & 7 & 84 & $\mathrm{Vb}$ & 99,75 & 15,75 \\
\hline $4 \mathrm{R}-1$ & 7 & 84 & $\mathrm{Va}$ & 90,78 & 6,78 \\
\hline $5 R-2$ & 2 & 111 & $\mathrm{Vc}$ & 152,25 & 41,25 \\
\hline $5 R-2$ & 2 & 111 & $\mathrm{Vb}$ & 147,37 & 36,37 \\
\hline $5 R-2$ & 2 & 111 & $\mathrm{Va}$ & 122,27 & 11,27 \\
\hline $5 R-2$ & 2 & 111 & $\mathrm{~J}$ & 170,50 & 59,50 \\
\hline $5 R-2$ & 3 & 188 & V & 212,66 & 24,66 \\
\hline $6 \mathrm{R}-2$ & 1 & 274 & V & 249,21 & 335,21 \\
\hline 7R-1 & 9 & 194 & $\mathrm{Va}$ & 114,76 & 280,76 \\
\hline $7 \mathrm{R}-1$ & 9 & 194 & MF & 340,9 & 146,9 \\
\hline $8 \mathrm{R}-1$ & 5 & 14 & V1 & 328,66 & 314,66 \\
\hline $8 \mathrm{R}-1$ & 5 & 14 & $\mathrm{CL}$ & 141,44 & 127,44 \\
\hline $8 \mathrm{R}-3$ & 1 & 348 & $\mathrm{PF}$ & 174,18 & 186,18 \\
\hline $8 R-3$ & 1 & 348 & Va & 192,32 & 204,32 \\
\hline $9 \mathrm{R}-1$ & 4 & 75 & CTV & 221,32 & 146,32 \\
\hline $9 \mathrm{R}-2$ & 8 & 295 & $\mathrm{Vb}$ & 160,73 & 225,73 \\
\hline $9 \mathrm{R}-2$ & 8 & 295 & CTV & 50,15 & 115,15 \\
\hline $9 \mathrm{R}-2$ & 8 & 295 & $\mathrm{PF}$ & 50,15 & 115,15 \\
\hline 9R-2 & 8 & 295 & Va & 325,12 & 30,12 \\
\hline $10 \mathrm{R}-1$ & $5 \mathrm{~A}$ & 269 & V4 & 0.68 & 91,68 \\
\hline 10R-1 & $5 \mathrm{~A}$ & 269 & MV & 7,68 & 98,68 \\
\hline $10 \mathrm{R}-1$ & $5 \mathrm{~A}$ & 269 & V3 & 326,75 & 57,75 \\
\hline $10 R-1$ & $5 A$ & 269 & $\mathrm{Pf}$ & 57,14 & 148,14 \\
\hline 10R-2 & 1 & 90 & V & 30,32 & 300,32 \\
\hline 10R-2 & $2 \mathrm{~A}$ & 8 & CTVb & 293,8 & 285,8 \\
\hline $10 \mathrm{R}-2$ & $2 \mathrm{~B}$ & 8 & CTV & 0,2 & 352,2 \\
\hline $10 \mathrm{R}-2$ & $2 B$ & 8 & PF & 247,8 & 239,8 \\
\hline
\end{tabular}

\begin{tabular}{|c|c|c|c|c|c|}
\hline $\begin{array}{l}\text { Core, } \\
\text { section }\end{array}$ & Piece & $\begin{array}{c}\text { Char. declin. } \\
\left(^{\circ}\right) \\
\text { (uncorr.) }\end{array}$ & Feature & $\begin{array}{c}\text { Strike, dip } \\
\left({ }^{\circ}\right) \\
\text { (uncorr.) }\end{array}$ & $\begin{array}{c}\text { Strike, dip } \\
\left({ }^{\circ}\right) \\
(\text { corr. })\end{array}$ \\
\hline 10R-2 & 2B & 8 & MF & 311,11 & 303,11 \\
\hline $11 \mathrm{R}-1$ & 7 & 239 & CTV & 214,40 & 335,40 \\
\hline $11 \mathrm{R}-\mathrm{I}$ & 7 & 239 & Bss & 214,40 & 335,40 \\
\hline $11 \mathrm{R}-1$ & 7 & 239 & PF & 214,40 & 335,40 \\
\hline $11 \mathrm{R}-1$ & 7 & 239 & $\mathrm{~V}$ & 214,40 & 335,40 \\
\hline $11 \mathrm{R}-1$ & 8 & 23 & SZ & 1170,60 & 147,60 \\
\hline $11 R-1$ & 8 & 23 & CTV & 183,30 & 160,30 \\
\hline $11 \mathrm{R}-1$ & 8 & 23 & PF & 183,30 & 160,30 \\
\hline 11R-2 & 5 & 8 & V & 60,263 & 52,26 \\
\hline $11 \mathrm{R}-2$ & 5 & 8 & V & 5170,11 & 162,11 \\
\hline $12 \mathrm{R}-1$ & 5 & 314 & IC & 0,54 & 46,54 \\
\hline $12 \mathrm{R}-1$ & 5 & 314 & Vd & 354,62 & 40,62 \\
\hline $12 \mathrm{R}-1$ & 5 & 314 & $\mathrm{Vb}$ & 29,24 & 75,24 \\
\hline $12 \mathrm{R}-1$ & 5 & 314 & Vc & 19,40 & 65,40 \\
\hline $12 \mathrm{R}-1$ & 5 & 314 & $\mathrm{Va}$ & 34,35 & 80,35 \\
\hline $12 \mathrm{R}-1$ & 5 & 314 & Vf & 333,70 & 19,70 \\
\hline $12 \mathrm{R}-2$ & 1 & 87 & PF & 180,40 & 93,40 \\
\hline $12 \mathrm{R}-2$ & 1 & 87 & V & 104,12 & 17,12 \\
\hline $12 \mathrm{R}-2$ & 1 & 87 & MVa & 214,40 & 127,40 \\
\hline $12 \mathrm{R}-2$ & 1 & 87 & $\mathrm{MVb}$ & 214,40 & 127,40 \\
\hline $12 \mathrm{R}-2$ & 2 & 171 & $\mathrm{MVa}$ & 195,73 & 24,73 \\
\hline $12 \mathrm{R}-2$ & 2 & 171 & $\mathrm{Va}$ & 195,73 & 24,73 \\
\hline $12 \mathrm{R}-2$ & 2 & 171 & $\mathrm{Vb}$ & 195,73 & 24,73 \\
\hline $13 R-1$ & 13 & 226 & $\mathrm{~V} 4 \mathrm{~b}$ & 188,53 & 322,53 \\
\hline $13 R-1$ & 13 & 226 & PF & 232,22 & 6,22 \\
\hline $13 \mathrm{R}-1$ & 13 & 226 & V4a & 108,25 & 242,25 \\
\hline $13 R-3$ & 2 & 184 & V & 141,76 & 317,76 \\
\hline $13 R-3$ & 6 & 24 & PF1 & 188,59 & 164,59 \\
\hline $13 R-3$ & 6 & 24 & PF2 & 205,48 & 181,48 \\
\hline $13 R-3$ & 6 & 24 & V & 148,91 & 24,9 \\
\hline $14 \mathrm{R}-1$ & 8 & 55 & V & 2,52 & 307,52 \\
\hline $14 \mathrm{R}-2$ & 1 & 157 & V & 186,60 & 29,60 \\
\hline $15 R-3$ & 1 & 151 & Vd & 335,58 & 184,58 \\
\hline $15 R-3$ & 1 & 151 & $\mathrm{Ve}$ & 166,71 & 15,71 \\
\hline $15 \mathrm{R}-3$ & 1 & 151 & $\mathrm{Va}$ & 33,29 & 242,29 \\
\hline $15 \mathrm{R}-3$ & 1 & 151 & $V_{c}$ & 8,70 & 217,70 \\
\hline $15 R-3$ & 1 & 151 & $\mathrm{Vb}$ & 168,63 & 17,63 \\
\hline $16 \mathrm{R}-2$ & 1 & 178 & $\mathrm{~V} 2 \mathrm{~b}$ & 255,11 & 77,11 \\
\hline $16 \mathrm{R}-2$ & 1 & 178 & SZ & 342,58 & 164,58 \\
\hline $16 \mathrm{R}-2$ & 1 & 178 & V4 & 90,90 & 272,90 \\
\hline $16 \mathrm{R}-2$ & 1 & 78 & V3 & 324,62 & 146,62 \\
\hline $16 \mathrm{R}-2$ & 4 & 41 & V4 & 151,76 & 110,76 \\
\hline $16 \mathrm{R}-3$ & 1 & 159 & V3 & 217,5 & 58,5 \\
\hline $16 \mathrm{R}-3$ & 5 & 162 & V & 198,24 & 36,24 \\
\hline $16 R-4$ & 7 & 135 & CTV & 328,33 & 193,33 \\
\hline $16 \mathrm{R}-4$ & 7 & 135 & F & 167,17 & 32,17 \\
\hline
\end{tabular}

Notes: The correction factor is equal to $360^{\circ}-\mathrm{D}$ (the uncorrected characteristic remanent declination). Therefore the corrected orientations are in the system where north is parallel to the remanent declincation. Abbreviations: $\mathrm{BS}=$ brittle shear zone; $\mathrm{CL}$ = compositional layering; $\mathrm{CTV}=$ composition or textural variation; $\mathrm{F}=$ fault; $\mathrm{IC}=$ igneous contact; $\mathrm{J}=$ joint; $\mathrm{MF}=$ magmatic fabric; $\mathrm{MV}=$ magmatic vein; $\mathrm{SZ}=$ shear zone; $\mathrm{PF}=$ plastic fabric; $\mathrm{V}^{*}=$ vein with number in the piece. Other abbreviations as in Table 2 . 